\title{
An electromagnetic arrayed pump to create arbitrary velocity profiles in fluid
}

\author{
Seyed Peyman Hashemi ${ }^{1} \cdot$ Mohammad Reza Karafi $^{1}$ [D $\cdot$ Mohammad Hossein Sadeghi ${ }^{1} \cdot$ Vahid Rezaei Esfedan $^{1}$
}

Received: 8 March 2021 / Accepted: 25 October 2021

Published online: 08 November 2021

(c) The Author(s) 2021 OPEN

\begin{abstract}
The present paper is conducted to develop a new structure of an electromagnetic pump capable of controlling the magnetic field in a rectangular channel. Common electromagnetic pumps do not create uniform velocity profiles in the cross-section of the channel. In these pumps, an M-shape profile is created since the fluid velocity in the vicinity of the walls is higher than that in its center. Herein, the arbitrary velocity profiles in the electromagnetic pump are generated by introducing an arrayed structure of the coils in the electromagnetic pump and implementing 3D numerical simulation in the finite element software COMSOL. The dimensions of the rectangular channel are $5.5 \times 150 \mathrm{~mm}^{2}$. Moreover, the magnetic field is provided using a core with an arrayed structure made of low-carbon iron, as well as five couples of coils. $20 \% \mathrm{NaoH}$ solution is utilized as the fluid (conductivity: $40 \mathrm{~S} / \mathrm{m}$ ). The arrayed pump is fabricated and experimentally created an arbitrary velocity profile. The pressure of the pump in every single array is $12 \mathrm{~Pa}$ and the flow rate is equal to $3375 \mathrm{~mm}^{3} / \mathrm{s}$. According to the results, there is a good agreement between the experimental test carried out herein and the simulated models.
\end{abstract}

\section{Article highlights}

- This is the first time that the idea of arrayed electromagnetic pump is presented. This pump uses a special arrayed core with coils; by controlling the current of each coil and the direction of the currents, the magnetic field under the core could be adjusted. By changing the magnetic field at any position in the width of the channel, the Lorentz force alters, which leads to different velocity and pressure profiles.

- Using COMSOL multiphysics software, the electromagnetic pump was simulated in real size compared to the

Keywords Arrayed magnetic pump $\cdot$ Velocity profile $\cdot$ Numerical solution

\author{
List of symbols \\ $J_{e}$ External current density, $A / m^{2}$ \\ $\mathrm{N}$ Number of windings \\ I Current, $A$
}

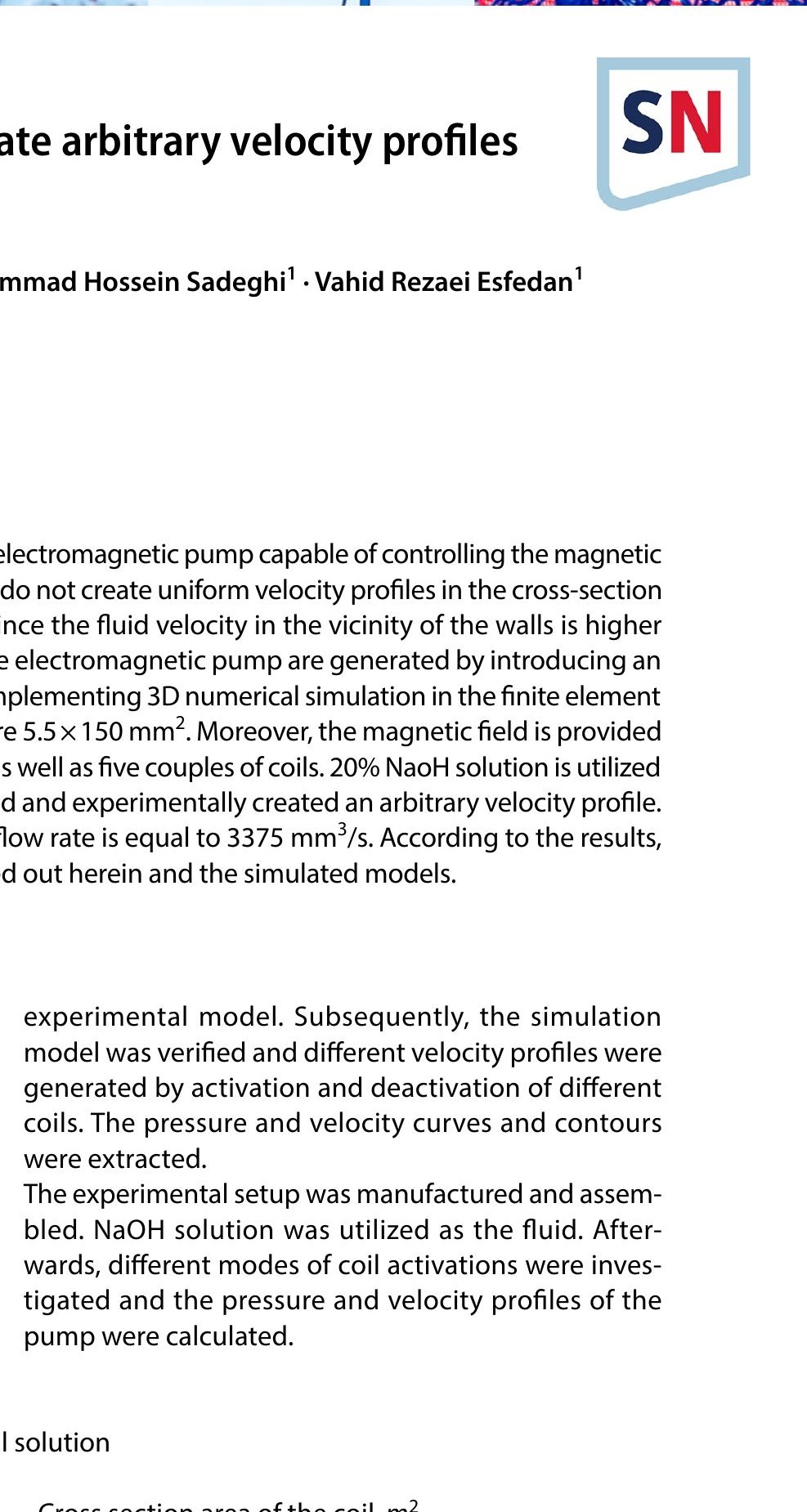

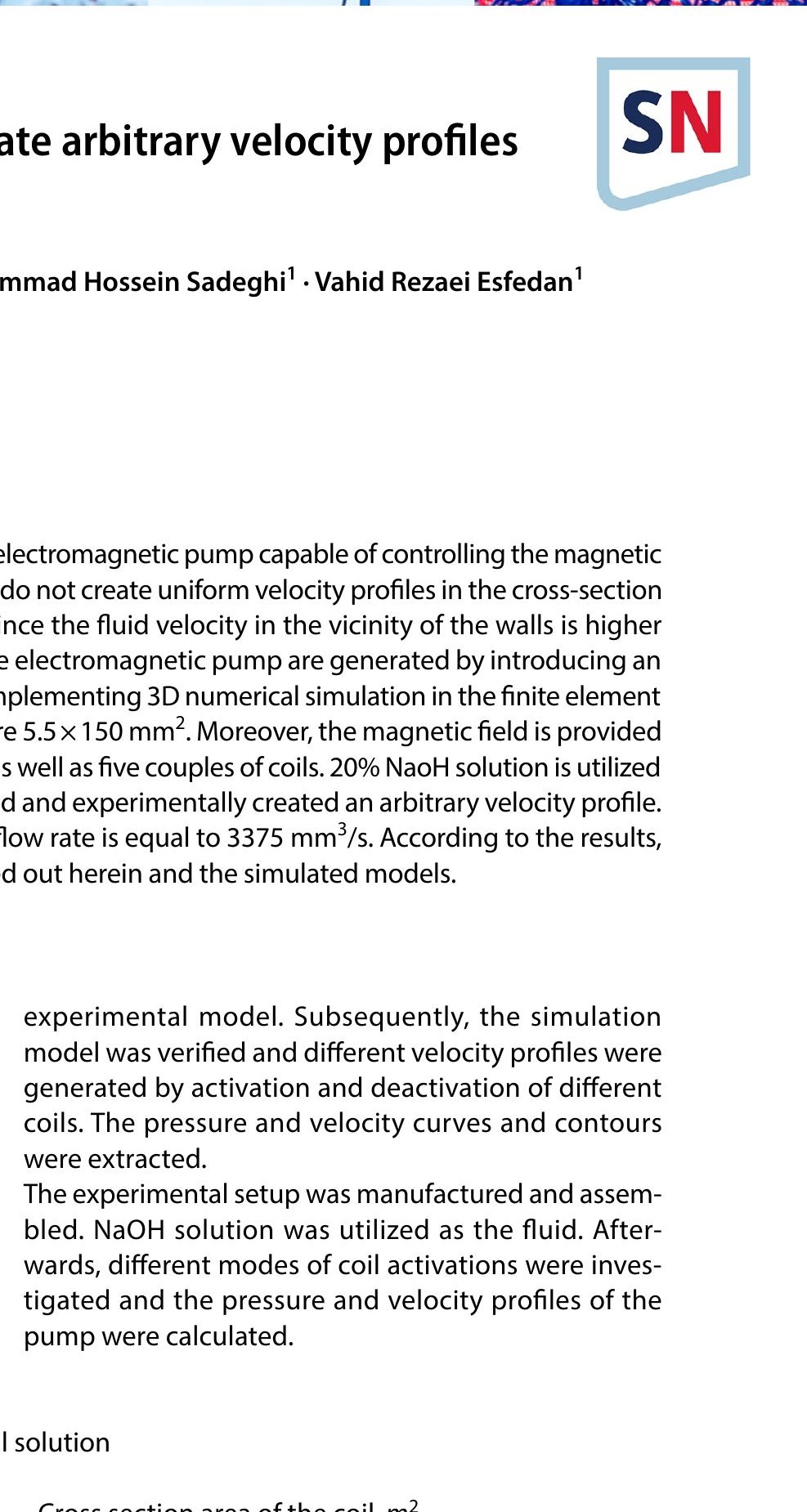

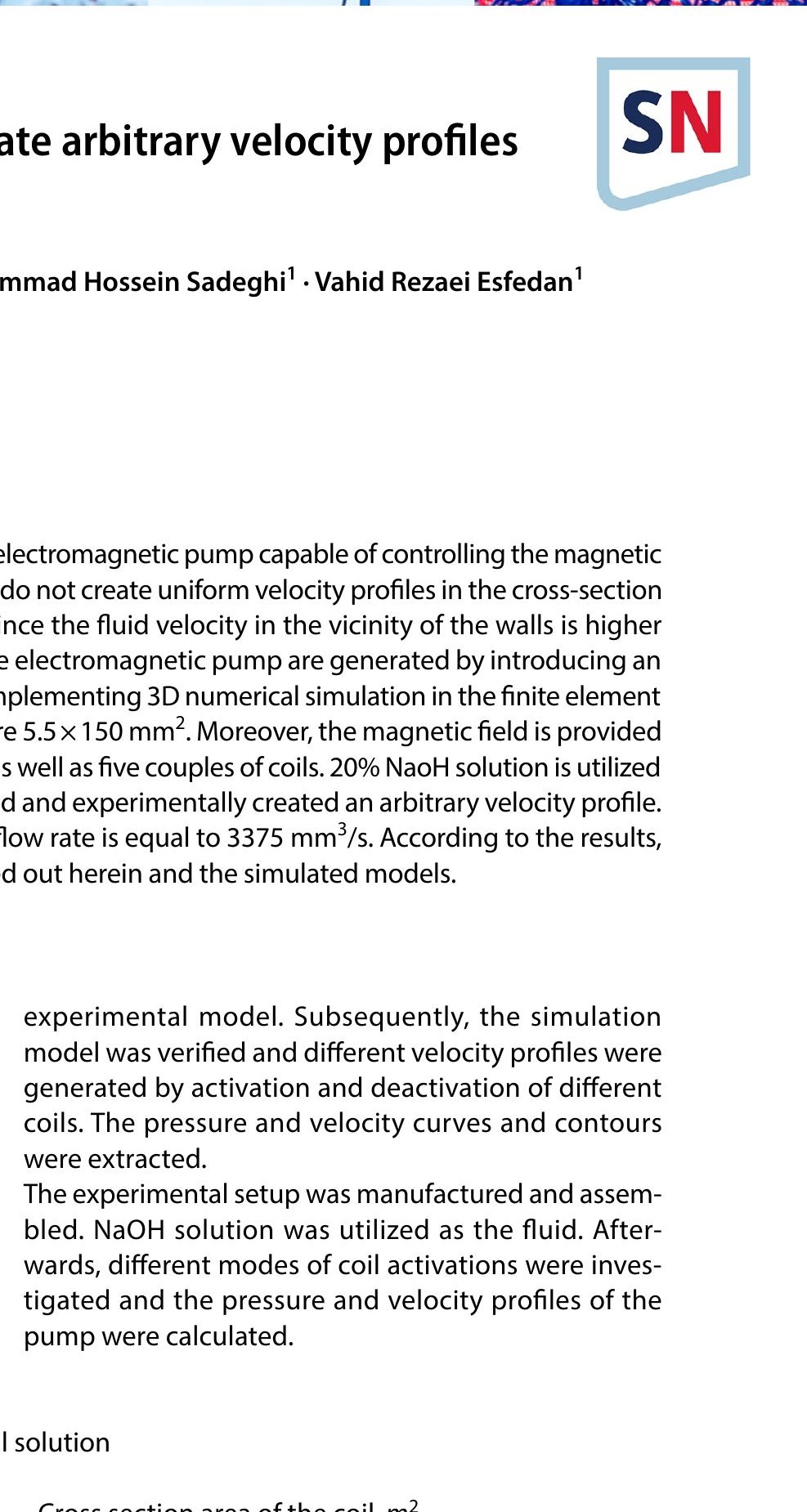

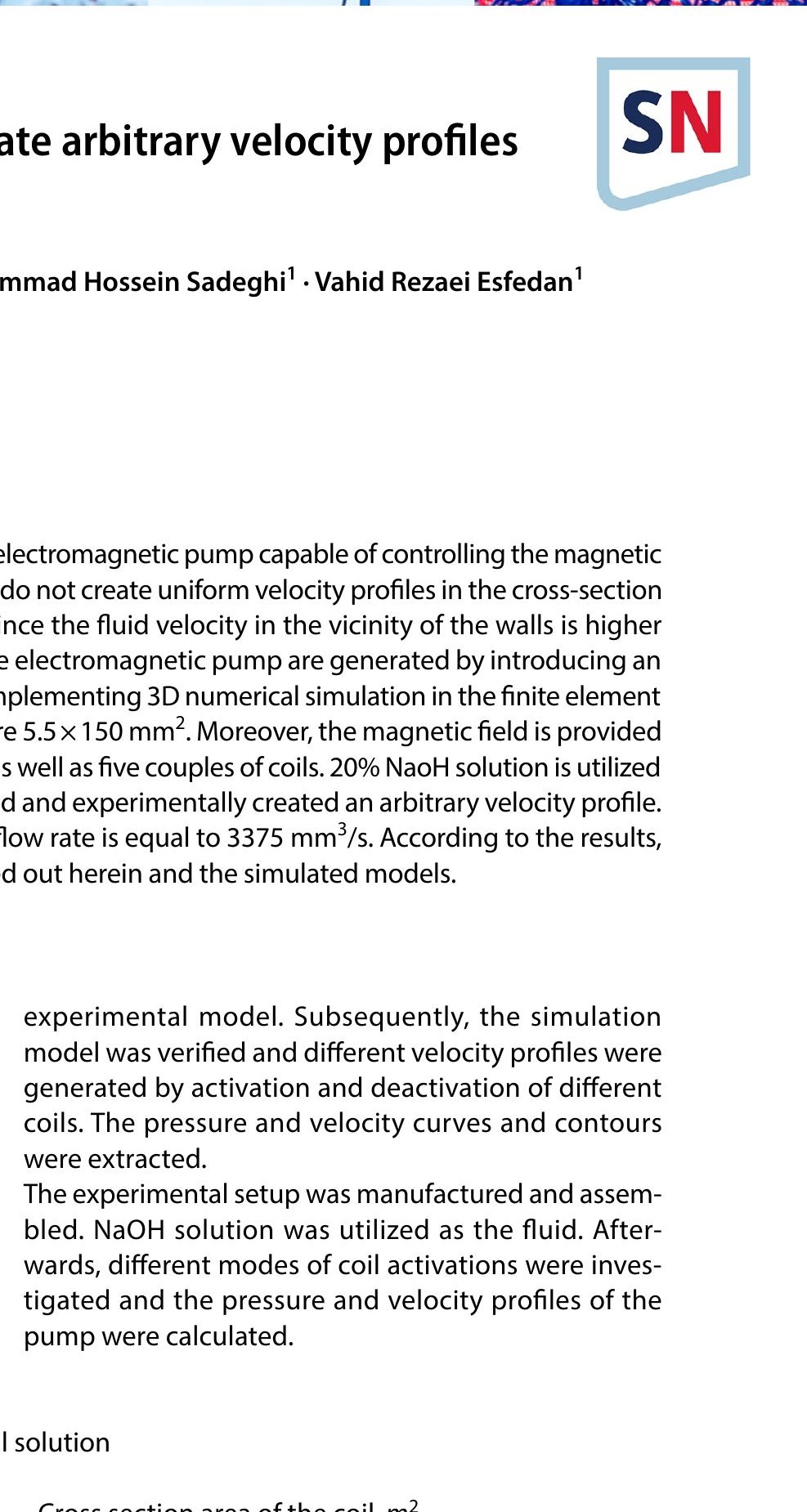

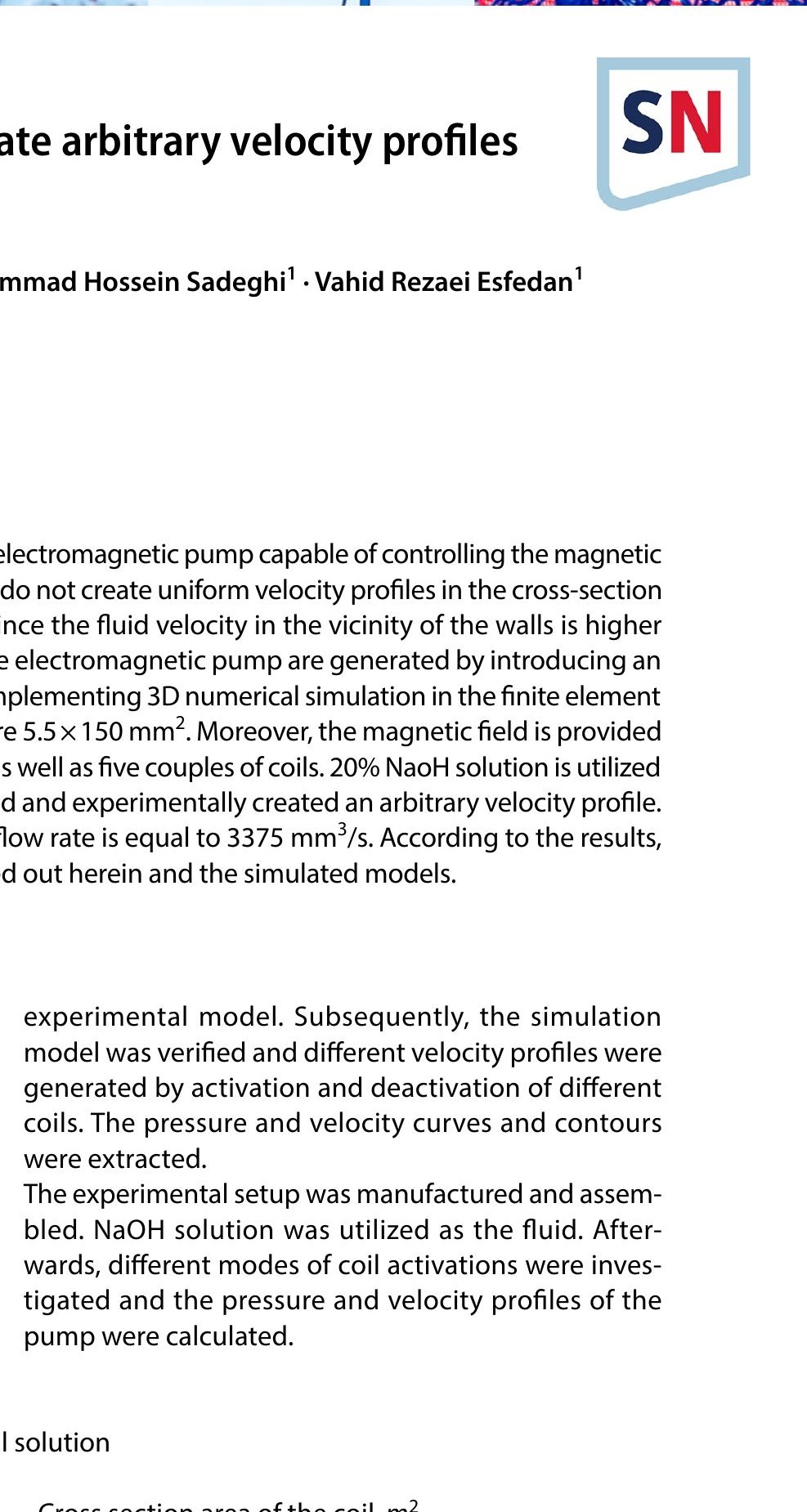

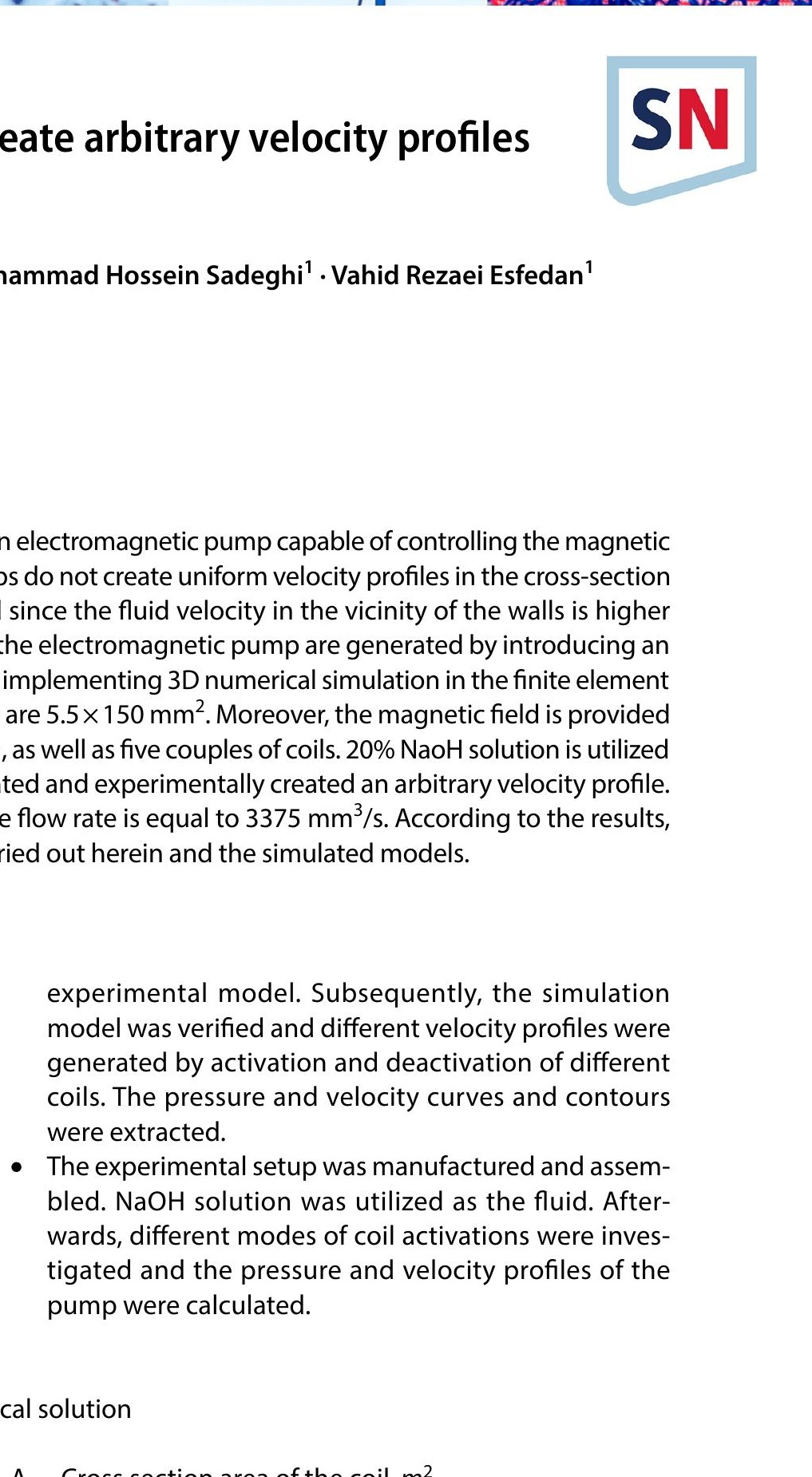

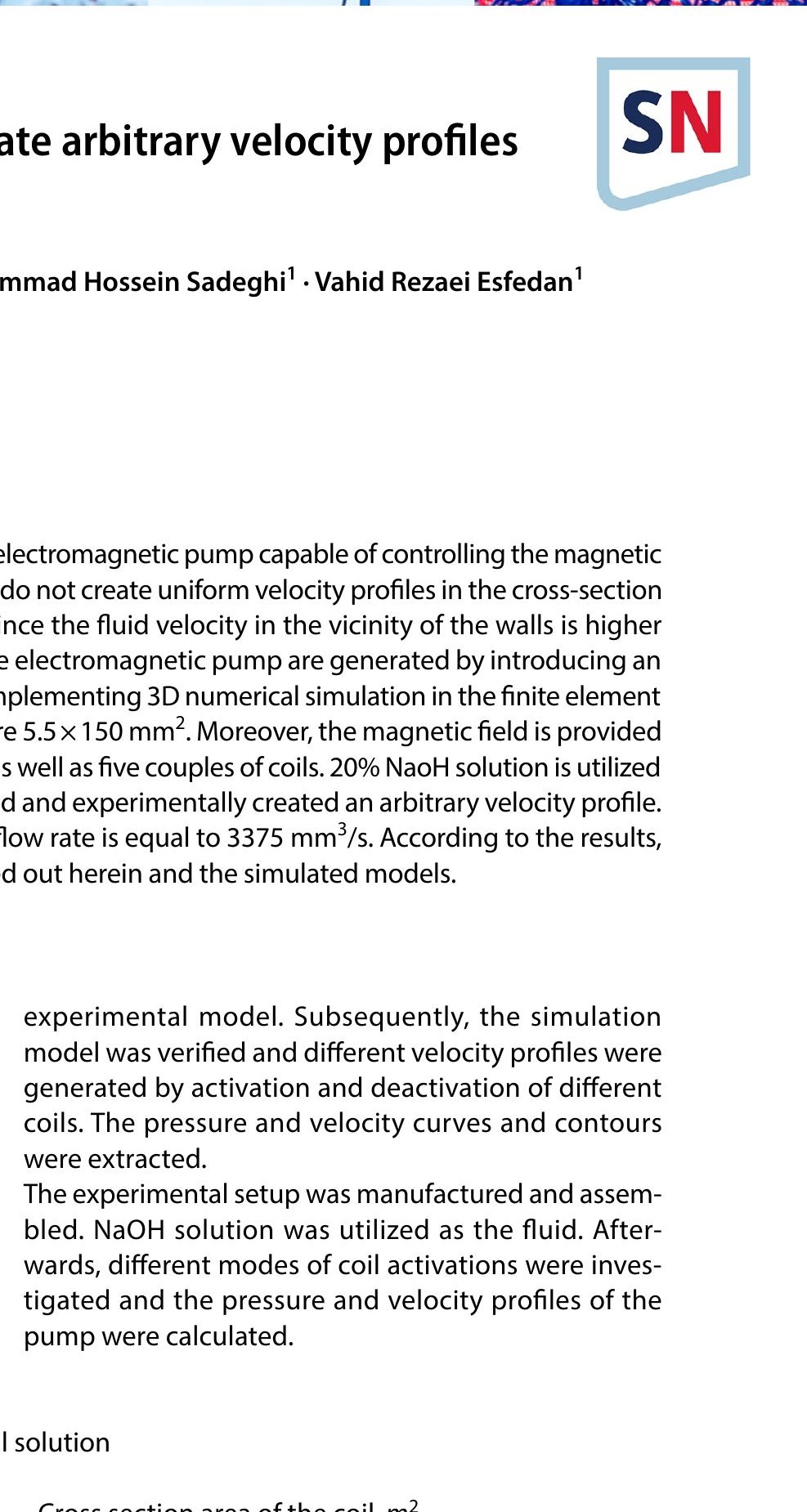

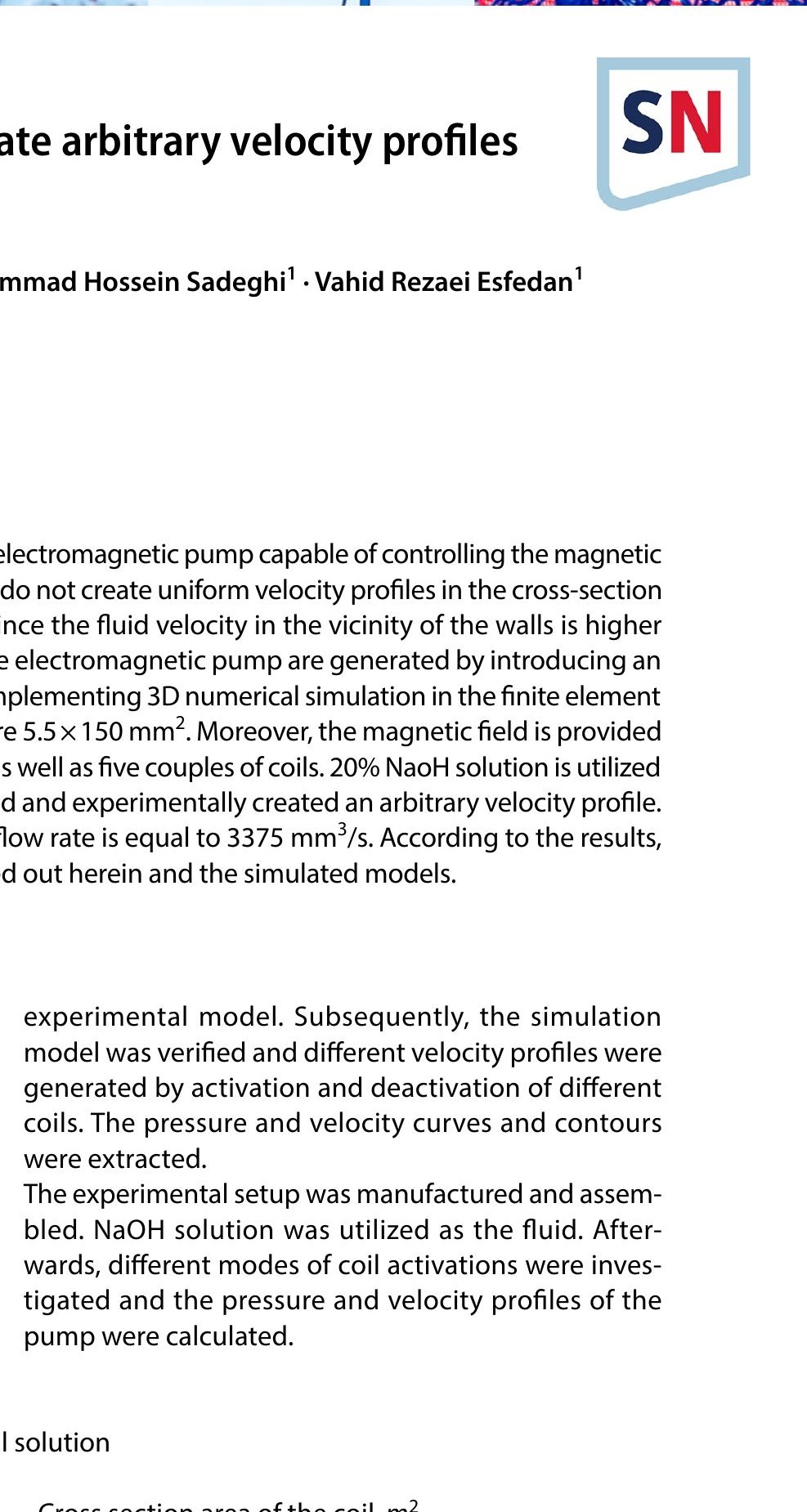

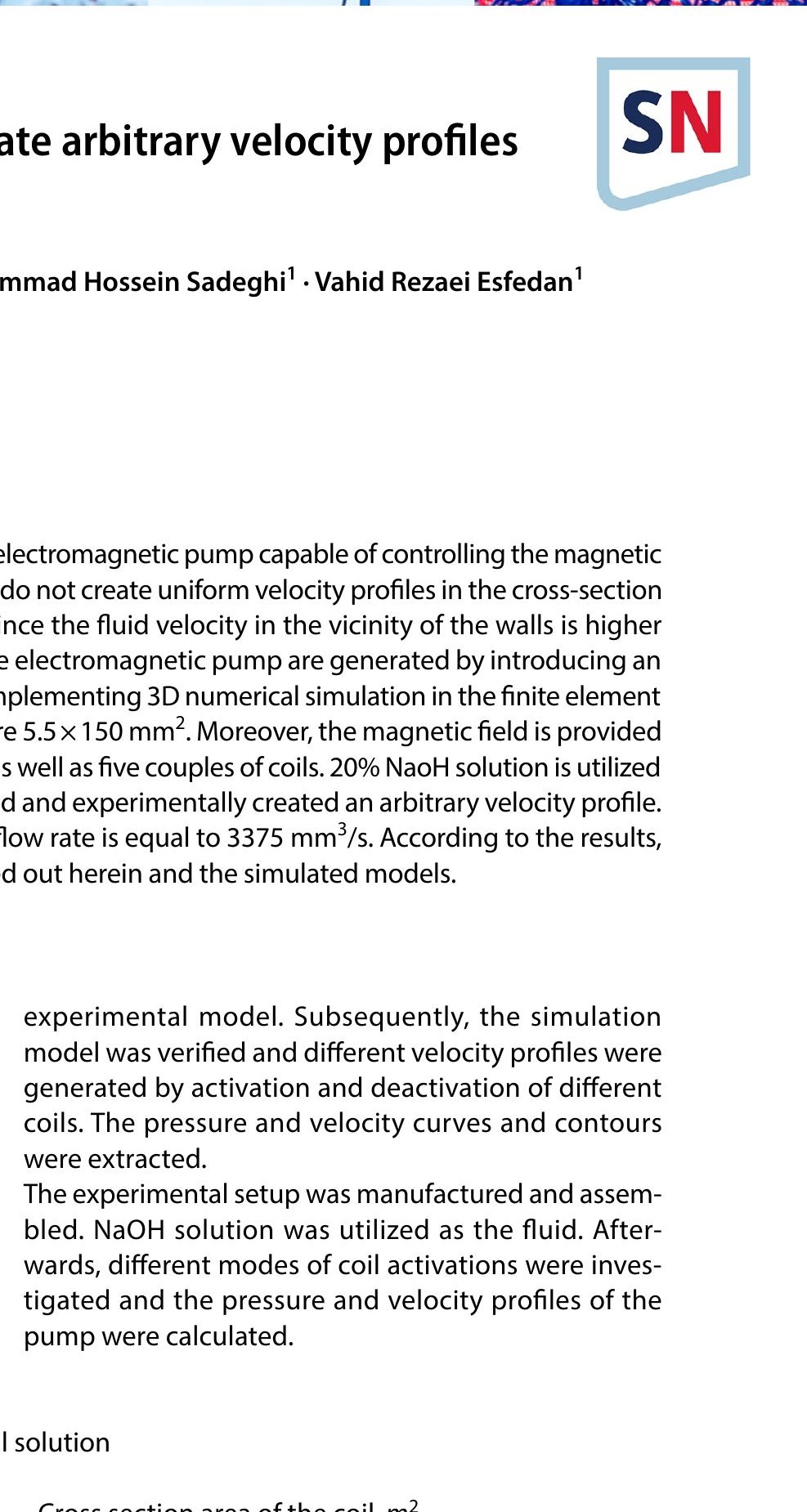

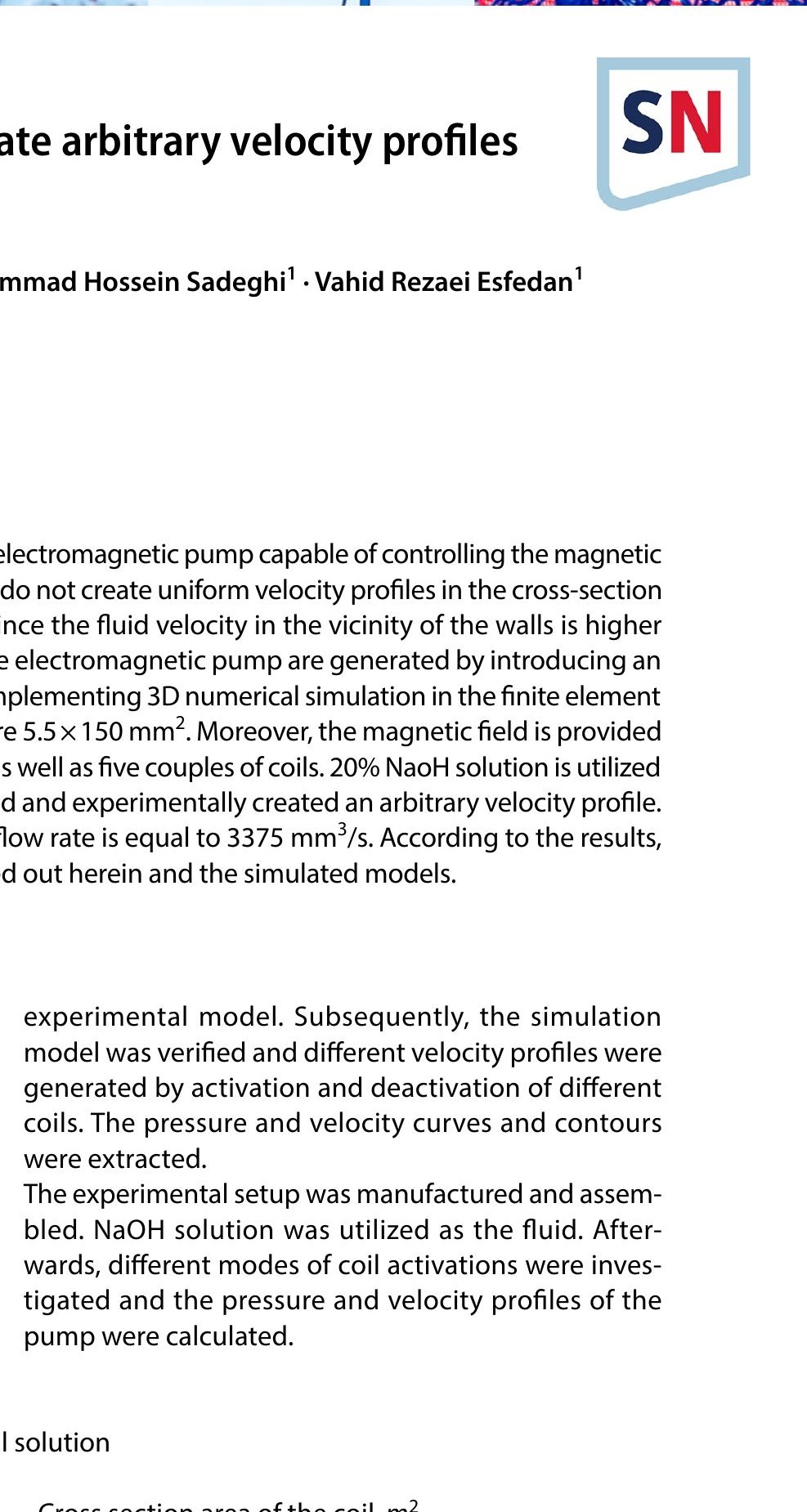

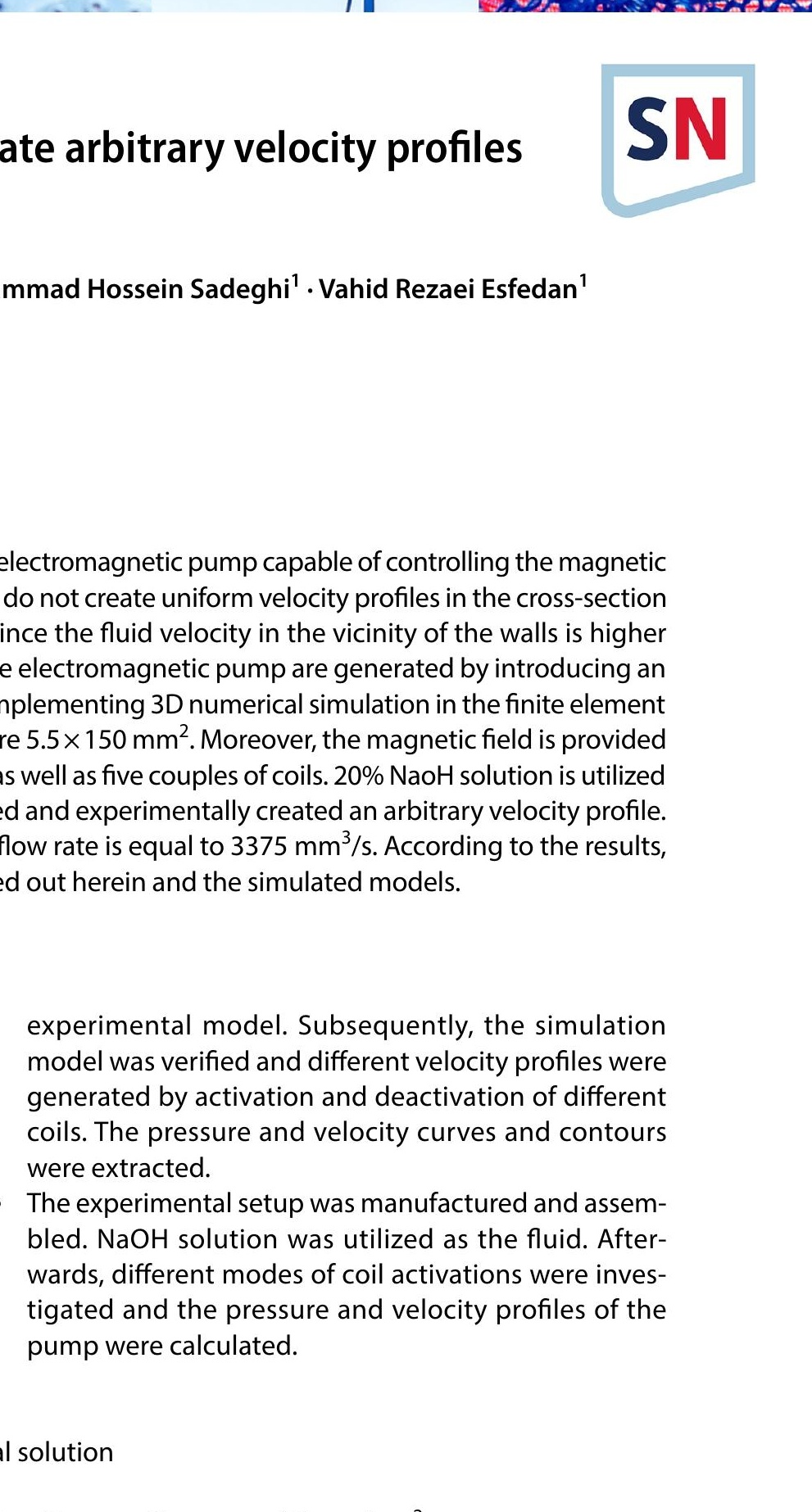

A Cross section area of the coil, $m^{2}$

$\mathrm{H}$ Magnetic field strength, $A / m$

$\vec{j}$ Current density vector, $A / m^{2}$

$\sigma \quad$ Electrical conductivity, $S / m$

Mohammad Reza Karafi, karafi@modares.ac.ir | ${ }^{1}$ Faculty of Mechanical Engineering, Tarbiat Modares University, Tehran, Iran.

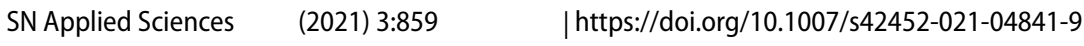


$\rho \quad$ Fluid density, $\mathrm{kg} / \mathrm{m}^{3}$

$\vec{B} \quad$ Magnetic flux density vector, $T$

$\vec{E} \quad$ Electric field vector, $V / m$

$\vec{u} \quad$ Fluid velocity vector, $\mathrm{m} / \mathrm{s}$

$\mathrm{p}$ Pressure, $p a$

$\vec{F}_{L} \quad$ Volumetric Lorentz force density vector, $N / \mathrm{m}^{3}$

g Earth gravity, $m / s^{2}$

$\checkmark$ Fluid volume, $\mathrm{mm}^{3}$

Q Volumetric flow rate, $\mathrm{mm}^{3} / \mathrm{s}$

t Time, s

\section{Introduction}

The fluid flow plays an enormous role in most industrial processes. Numerous pumps have been employed in industry, among which electromagnetic pumps have a long life and less leakage owing to their non-contact and absence of moving components [1]. In electromagnetic pumps, a Lorentz force in the direction perpendicular to the current and the magnetic field is created by passing an electric current through a conductive fluid, such as saltwater, and by concurrently applying a high-intensity magnetic field perpendicular to the electric current. The idea of using an electromagnetic pump for the cooling system of the nuclear reactor in order to move molten sodium fluid was established in 1960. This idea was developed in 1970 and different types of electromagnetic pumps were utilized in foundry industries and melt pumping of electricityconductor metals [2]. The alternating current (AC) pumps have higher efficiency in comparison with the direct current (DC) ones; however, their manufacturing cost and maintenance are higher [3]. Common electromagnetic pumps do not make uniform velocity profiles; the fluid velocity in the vicinity of the walls is higher than that in its center. Accordingly, they create an M-shape velocity profile in the cross-section of the channel. Several numerical solution approaches were developed over the 20 recent years; for example, in 1991, Ramos et al. [4] used a numerical method to study the fluid flow in the electromagnetic pump. In their research, the fluid flow was a function of the Reynolds number, electrode length, and conductivity of the walls. The fluid velocity profile was obtained in the form of an M-shape velocity profile, which got more intense with the increase in the Reynolds number and electrode length. Furthermore, Hughes investigated the fluid flow in microscopic dimensions [5]. Andreev et al. studied fluid flow under the influence of a heterogeneous magnetic field two-dimensionally; in their research, the Hartmann number was 400 and the Reynolds number was considered between 500 and 16,000. Eventually, they identified three turbulent areas, namely a turbulence suppression region, a vertical region, and a wall jet region [6]. Additionally, a 3D simulation of planar flow was carried out by Votyakov and his result was compared to the experimental ones [7]. Daoud et al. also presented a 3D simulation of a DC electromagnetic pump for aluminum melt with a high Reynolds number and non-uniform magnetic field. They used two permanent magnets at the upper and lower parts of the rectangular channel to create the magnetic field. Moreover, they simulated an electromagnetic pump which acted as a pump and a brake. In the brake status, the magnetic direction and applying fluid flow are in a way that the Lorentz force is applied on the opposite direction of the fluid movement [8]. Aoka et al. in 2013, experimentally and numerically studied the electromagnetic pump with permanent magnet and electrolyte fluid. They used COMSOL for solving the Navier-Stokes equations coupled with the Maxwell equations $[9,10]$. Jamalabadi et al. analytically investigated the effect of magnetic and electrical angular frequency on the velocity distribution in a magneto-hydro-dynamic pump as a ship propulsion system [11]. Dong et al. developed electromagnetic transport (EMT) process with plane induction electromagnetic pump (EMP) for transporting liquid aluminum alloy during casting. They employed the numerical model for analyzing the parameter of the rectangular EMP on EMT performance and showed that the irregular outflow is unfavorable to the precise control of EMT process and that the air entrapment would cause oxidation and is harmful to the quality of casting [12-14]. In 2015, Abdullina et al. simulated the liquid metal flow in an electromagnetic field. They used ANSYS for numerical calculations [15]. In 2016, Derakhshan et al. examined the relationships governing electromagnetic micropumps. In microdimensions, they studied the effect of different parameters, including channel height, magnetic field size, and electrode current on the pump performance. Their investigation was carried out experimentally and numerically; the experimental results and simulation were then compared [16]. The majority of the recent research in the field of the electromagnetic pump are related to the cooling cycle of the nuclear power plants. Sodium molten metal has been utilized in the cooling cycle owing to its features. Due to its high temperature and special conditions, molten sodium is displaced by employing an electromagnetic pump. Geza et al. numerically simulated a large electromagnetic pump used in a power plant with sodium, as the coolant liquid [17]. Kim et al. successfully moved molten sodium in a $64 \mathrm{~cm}$ diameter channel with an electromagnetic pump having a flow rate of $0.86 \mathrm{~m}^{3} / \mathrm{s}$ and optimized its hydraulic parameters. Their pump was of the circular linear arrangement, which was stimulated by an alternating current source [18]. In another work, they numerically and experimentally scrutinized a DC electromagnetic pump with permanent 
magnets in a rectangular channel. Their fluid was molten lithium. To achieve the maximum pressure (1.5 MPa), they optimized electric parameters and pump dimensions at $200^{\circ} \mathrm{C}$ using a numerical method [19]. Wang et al. experimentally created a closed cycle by the means of permanent magnets; these magnets applied force to the fluid in two stages. The thickness of the channel was $4 \mathrm{~mm}$. The instantaneous temperature of the fluid was also measured via a thermocouple. They simulated the effect of the twostage force applied to the fluid on the metal temperature in COMSOL and studied its performance in the cooling of electronic components. Their results indicated that the applied two-stage force contributed to improving the cooling performance [20]. Zakeri et al. in 2019, used finite volume method and numerically studied the effect of geometrical parameters, such as depth, width, and length of the side electrodes, on micro pump performances with a rectangular channel. They showed that a wider channel with long electrodes could be used in favor of high performance for high flow rates, high pressure, and energyefficient demands [21]. In 2020, Lee et al. numerically investigated the effect of flow velocity, applied voltage, magnetic flux density, Hartman number, and so forth on MHD pump performance in microchannel cooling system for heat dissipating element with different nanofluids [22]. Klüber et al. also presented a 3D simulation model to investigate the transition of turbulent hydrodynamic liquid metal flow towards a steady-state laminar MHD flow in a circular section. They reported the velocity profiles in different positions under a strong magnetic field [23]. In 2020 , Zhang et al. introduced a novel layered structure of an electromagnetic pump in order to displace the molten metal. They achieved an optimal structure of the layered pump by simulating it in COMSOL. They utilized a DC pump and their optimal channel structure consisted of five electromagnets and a continuous iron yoke. To increase the pressure of the pump, the channel height was considered $1.4 \mathrm{~mm}$. In their research, the working temperature was lower than $120^{\circ} \mathrm{C}$ [24].

In all the investigations conducted to date, the magnetic field across the channel in the electromagnetic pump has been considered to be uniform; as mentioned previously, one of the most common problems of electromagnetic pumps is the lack of uniform distribution of the velocity profile, particularly in liquid metal castings in which irregular outflow would cause serious problems in the quality of the casting pieces. The current paper aimed to investigate the feasibility of using a different magnetic fields in various areas in order to control the velocity profile. In Sect. 3 of this paper, the structure of the arrayed pump is introduced and the assembled experimental setup is shown. A DC electromagnetic pump with an arrayed magnetic field consisting of five couples of coils was designed and manufactured. Section 4 explains how $\mathrm{NaOH}$ solution was used as the fluid and simulation of the pump and fluid was addressed in the finite element software COMSOL. In Sects. 5 and 6, the possibility of creating an arbitrary velocity profile in the arrayed electromagnetic pump is evaluated experimentally and with a simulation approach by activating and deactivating the coils.

\section{Principles and equations of electromagnetic pumps}

The performance principles of the electromagnetic pumps follow the Faraday induction law: if an electric conductor is placed in a flow field and has an electric current perpendicular to the magnetic field, a force is imposed to the conductor whose direction is perpendicular to the field and current. Figure 1 depicts the schematic of the electromagnetic pump.

The formulation of the steady state magneto hydrodynamic 3D model has been derived from the Maxwell equations (electromagnetic part) and the Ohm's law for moving medium coupled with the Navier-Stokes equation and Continuity equation (fluid dynamics part). The governing equations solved by $\mathrm{COMSOL}$ in the numerical simulation could be summarized as follows:

Electromagnetic part:

The current density of coils is calculated as follows:

$J_{e}=\frac{\mathrm{NI}_{\text {coil }}}{\mathrm{A}}$

Ampere law is used to calculate magnetic field:

$\nabla \times \mathrm{H}=\mathrm{J}$

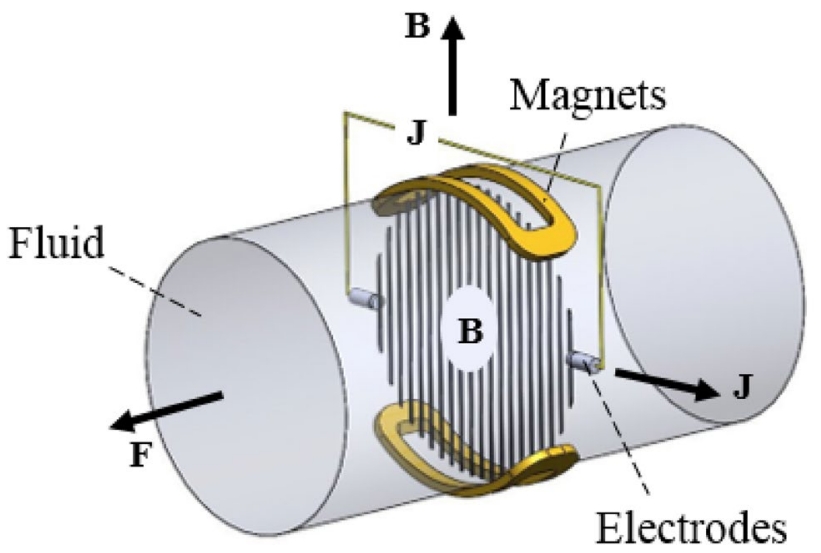

Fig. 1 The structure of the electromagnetic pump 
Ohm's law is used to modify the current density:

$\vec{\jmath}=\sigma(\vec{E}+\vec{u} \times \vec{B})$

Fluid dynamics part (Laminar flow model):

Navier-Stokes equation is employed to calculate the velocity of the fluid:

$\rho(\overrightarrow{\mathrm{u}} \cdot \nabla) \overrightarrow{\mathrm{u}}=\nabla \cdot\left[-\mathrm{pl}+\mu\left(\nabla \overrightarrow{\mathrm{u}}+(\nabla \overrightarrow{\mathrm{u}})^{\top}\right)\right]+\overrightarrow{\mathrm{F}_{\mathrm{L}}}$

Incompressibility conditions of the fluid is used to solve the equation:

$\rho \nabla \cdot(\overrightarrow{\mathrm{u}})=0$

Volumetric Lorentz force is calculated as follows:

$\overrightarrow{\mathrm{F}}_{\mathrm{L}}=\overrightarrow{\mathrm{J}} \times \overrightarrow{\mathrm{B}}$

where $\sigma, \rho, \mu, \mathrm{B}, \mathrm{J}, \mathrm{E}, \mathrm{u}$, and $\mathrm{P}$ are electrical conductivity, fluid density, dynamic viscosity, magnetic flux density vector, current density vector, electric field vector, fluid velocity vector, and pressure, respectively. The vectors of the magnetic flow density and current density are considered as follows:

$\vec{B}=B_{x} \hat{i}+B_{y} \hat{j}+B_{z} \hat{k}$

$\vec{J}=J_{x} \hat{i}+J_{y} \hat{j}+J_{z} \hat{k}$

According to Relation (6), the volumetric force components are equal to:

$F_{x}=J_{y} B_{z}-B_{y} J_{z}$

$F_{y}=J_{z} B_{x}-B_{z} J_{x}$
$F_{z}=J_{x} B_{y}-B_{x} J_{y}$

The volumetric force components drive the fluid in the channel. In the simulation by COMSOL, these relations are defined in the laminar flow physic. By coupling the three different physics in COMSOL (Electromagnetic, DC conductive media, and laminar flow), the simulation can be carried out. The problem is solved using a stationary segregated solver.

\section{The structure of the arrayed electromagnetic pump}

In the electromagnetic pump, the magnetic field is uniformly distributed across the fluid channel using the coil. Moreover, there is a slight difference in the density of the magnetic field. Meanwhile, in the current paper, the goal is to apply different magnetic fields in the direction of the channel width and the capability of controlling the density of the magnetic field in different sections. For this purpose, an arrayed structure was designed which creates a variable and controllable magnetic field. Figure 2 represents the schematic of the core of the arrayed coil. The arrayed magnetic core consisted of five partitions which could apply different magnetic field from $B_{1}$ to $B_{5}$. By applying the magnetic field (the blue highlighted arrows) in the $-Y$ direction and the current (the green highlighted arrows) in $+X$ direction, the movement of the fluid (the red highlighted arrows) would be toward the inside of the plane.

Based on Fig. 2, by activating each couple of the coils, the magnetic field is centrally created in that area of the channel. It should be mentioned that a different field was created by applying various currents in every single coil. If the directions of the magnetic and electric fields are from top to bottom and right to left, respectively, given the right-hand rule according to Eq. 6, the movement
Fig. 2 The schematic and dimension (in $\mathrm{mm}$ ) of the core and the channel of a magnetic arrayed pump

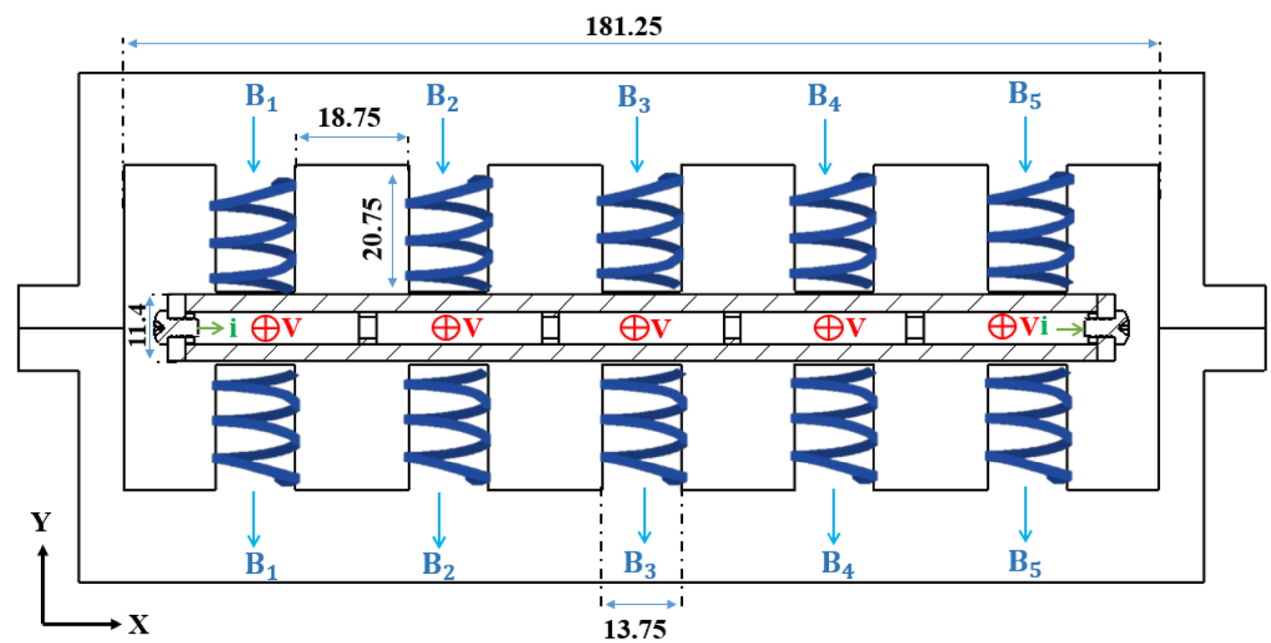




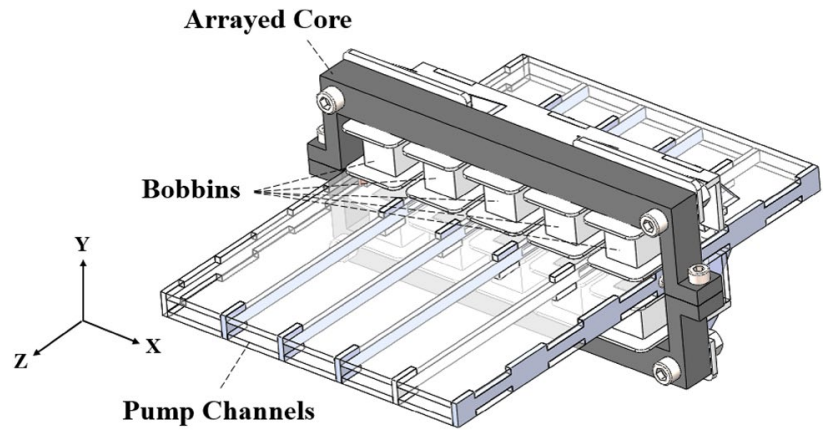

Fig. 3 The assembled view of the pump

Table $1 \mathrm{NaOH}$ characteristics

\begin{tabular}{lll}
\hline Parameter & Value & Unit \\
\hline Relative permittivity $\left(\varepsilon_{\mathrm{r}}\right)$ & 10 & - \\
Electric conductivity & 4 & $\mathrm{~S} / \mathrm{m}$ \\
Density & 1000 & $\mathrm{~kg} / \mathrm{m}^{3}$ \\
Dynamic viscosity & $1.2 \mathrm{e}^{-3}$ & $\mathrm{~Pa} . \mathrm{s}$ \\
Relative permeability & 1 & - \\
Temperature & 25 & ${ }^{\circ} \mathrm{C}$ \\
\hline
\end{tabular}

direction of the fluid and the applying of the Lorentz force will be perpendicular and inwards to the plane. The dimensions of the core were determined considering the bobbin dimensions of the coils. The width and height of the channel were $150 \mathrm{~mm}$ and $5.5 \mathrm{~mm}$, respectively; the number of the coils was five couples. Therefore, according to the channel thickness, the final dimensions of the core were based on Fig. 2. As shown, the pump channel is in the form of separated channels and to make sure that the current circuit is closed, it is embedded as grooves under the electromagnets. Figure 3 shows the assembled view of the arrayed pump.

The electrodes were made of copper. The characteristics of the $\mathrm{NaOH}$ saltwater defined in the software are presented in Table 1. Conductivity changes with the alterations in the concentration of solution and in this research, $20 \% \mathrm{NaOH}$ solution was used, which had the highest conductivity in water $\mathrm{NaOH}$ solution [25]. The pump channel was made with a $2.8 \mathrm{~mm}$ thick Plexiglas; the core material was low-carbon iron as well. The coils consisted of 130 rounds of $0.5 \mathrm{~mm}$ diameter copper wire. A DC power supply was employed for coils activation; in addition, the electric field was provided by a 180 -amp DC inverter device.

Figure 4 illustrates the assembled view of the pump and reservoir. As shown, the experimental setup is mounted in an inclined surface to guarantee a constant feed of fluid under the pump. The reservoir is filled with saltwater up to a height where the fluid is below the magnets.

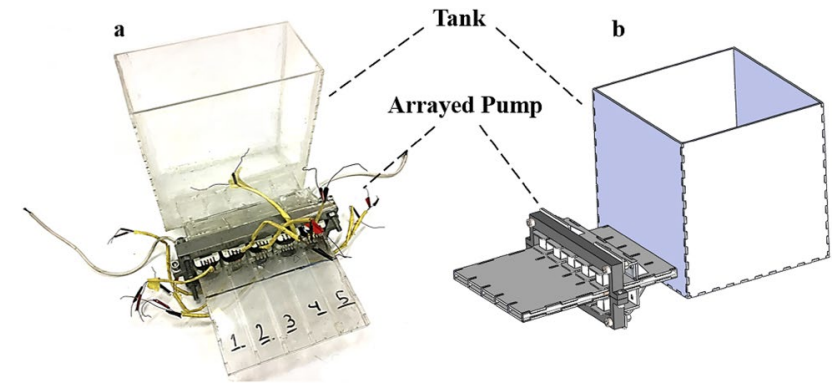

Fig. 4 The assembled view of the electromagnetic pump test track

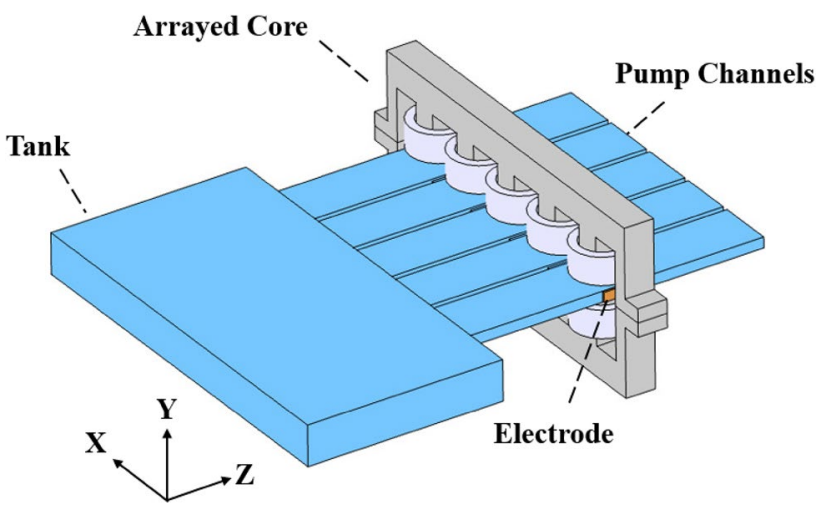

Fig. 5 The schematic of the pump modeled in COMSOL software

\section{Numerical simulation of arrayed electromagnetic pump}

According to the multiphysics nature of the rules governing the pump, the electricity, magnetic, and fluid flow phenomena should be combined to simulate function of the electromagnetic pump. For this purpose, the multiphysics software COMSOL, which is able to solve different physics and their combinations, was employed. The schematic of the pump modeled in COMSOL is illustrated in Fig. 5. The domain of the flow is given by a tank attached to arrays of rectangular flat channels. For the laminar fluid model, the inlet and outlet boundary conditions of the rectangular channel were determined by the inlet pressure Pinlet $=0$ and the end of the channels were closed. Incompressible flow was assumed and "Noslip" velocity conditions were considered on the sides, top, and bottom walls of the channels and the tank. The end of each channel is defined as the walls to identify the blocking end. The arrayed core, coils, the number of turns in the coils, wire diameters, and current passing through them were defined in magnetic fields physics. In the experimental and simulation conditions, there were 130 turns of $0.5 \mathrm{~mm}$ diameter wire in the coils and the 
Table 2 The number of elements for the four different meshes used to study mesh convergence

\begin{tabular}{lllll}
\hline & Mesh1 & Mesh2 & Mesh3 & Mesh4 \\
\hline Resolution & Normal & Fine & Finer & EXTRA fine \\
Elements & 34,915 & 70,262 & 293,859 & 655,477 \\
\hline
\end{tabular}

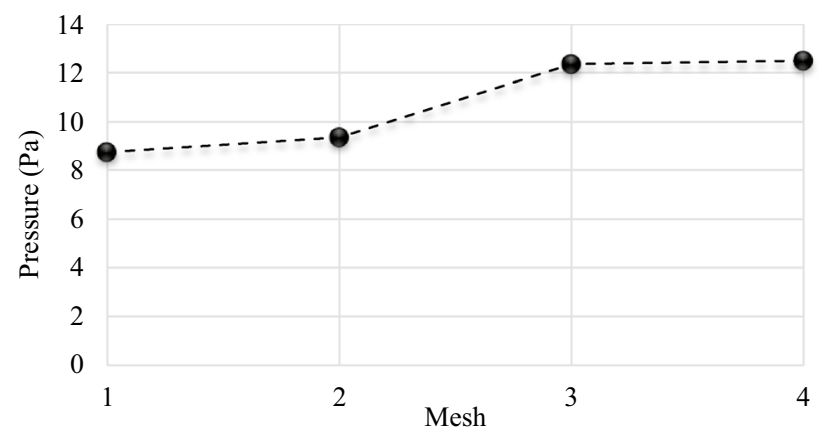

Fig. 6 Mesh convergence plot of the pressure

passing current was also equal to $3 \mathrm{amp}$. Afterwards, by enabling and disabling the coils domains in this physic, the effect of activation and deactivation of the coils in the pump was investigated.

The current passing through a pump was defined in electricity physics. In this case, one electrode was defined as the terminal (the current is defined in this electrode) and the other one played the role of the ground. Initially, the magnetic physics and electricity were activated and afterwards, the Lorentz force was applied in the form of a volumetric force in the fluid physics after applying the magnetic field and current. The mesh convergence analysis was performed to ensure that the results are converged solutions. To do so, the pressure of the pump was considered as the output to compare the results in different mesh sizes. Four meshes were compared to different refinements. Table 2 shows mesh types and the number of used elements.

All the boundary conditions, like activated coils, coils current, and electrode currents, in the simulation were kept constant and only the mesh size changed. The mesh convergence could be seen in Fig. 6 .

As could be seen in Fig. 6 , the pressure of the pump increases with the rise in the mesh resolution and its constant in Mesh3. Therefore, Mesh3 was considered to be refined enough to resolve the operations of the pump sufficiently; this mesh was used for the rest of the simulations. Figure 7 shows the meshing view of the model. Another way to analyze the mesh is considering the minimum element quality of the mesh in Statistic tab in COMSOL, which should be greater than 0.1 [26]. The meshing minimum quality was 0.15 with the elements number of $2,938,569$.

\section{Numerical results}

In this section, the effect of activating and deactivating the electromagnets on the output velocity (in the form of the velocity contour), pressure, and the fluid velocity diagram along the axial line of the electrodes are explained. Figure 8 exhibits the axial line passing through the electrodes, which is the evaluation location of the velocity. Because of the low conductivity of the saltwater, the Reynolds number in this simulation was below 500 and was simulated in a laminar flow physic in COMSOL.

Figure 9 demonstrates the magnetic field variations in the direction of the channel width once the middle electromagnet (number 3 ) is active. As could be seen, the magnetic field increased only in the middle part. The dashed vertical lines on the graph illustrate the wall of each single core. The magnetic field passed merely through the active electromagnet and the pump channel and traveled the path along the core.

In the simulation, the maximum pressure produced by the pump was identified through blocking the end of the
Fig. 7 The meshing view of the model and number and the type of the elements
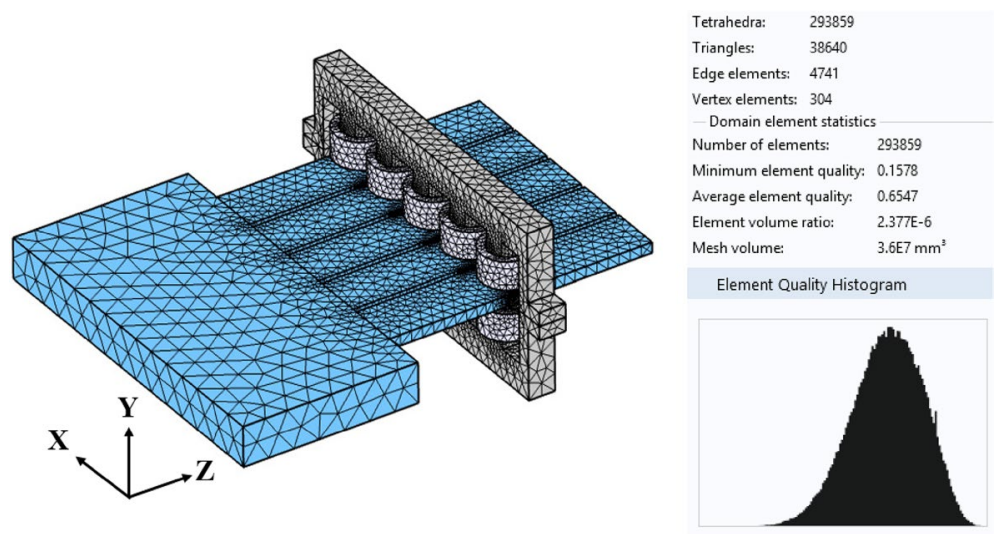


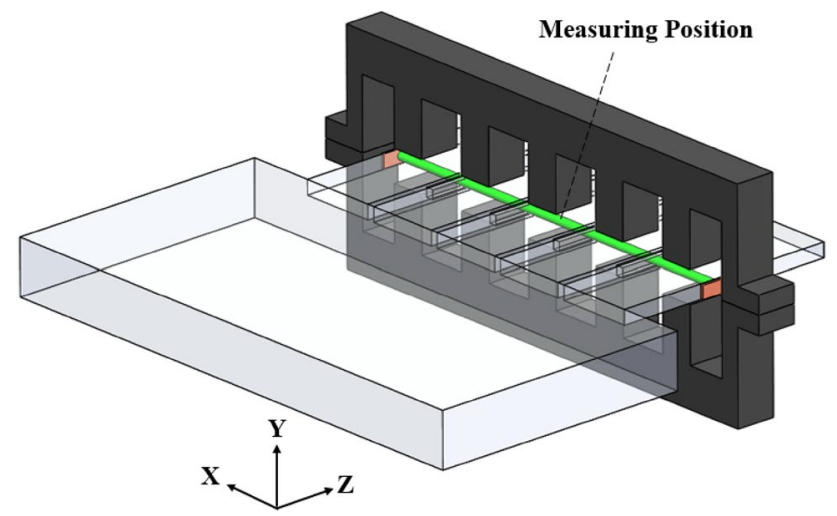

Fig. 8 The axial line of the electrodes (the evaluation location of the fluid velocity)
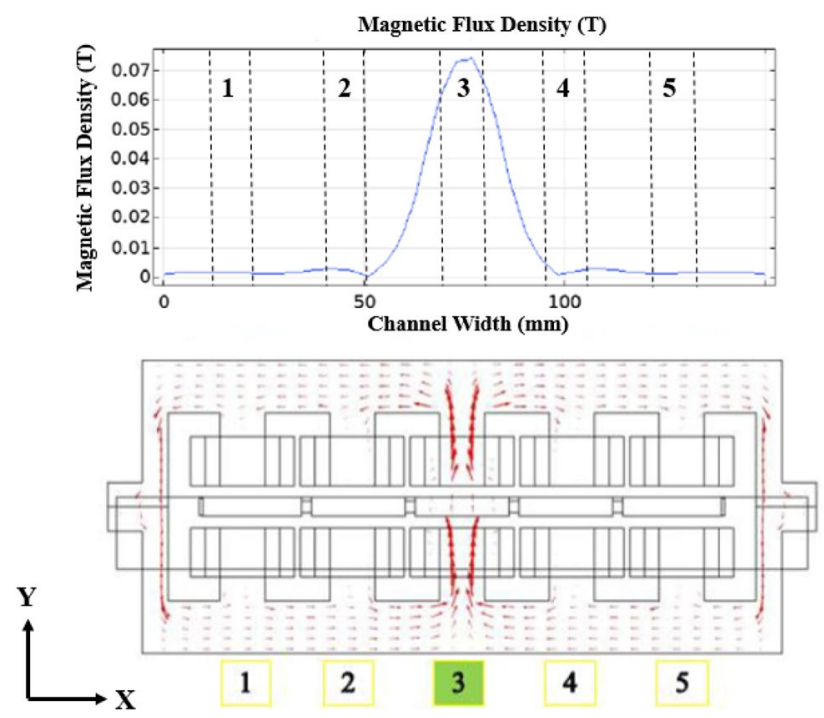

Fig. 9 The magnetic field profiles and lines once the middle coil was active

channel. As shown in Fig. 10, the maximum pressure was equal to $12 \mathrm{~Pa}$ at the front part of the pump. It suggested that adjacent channels are slightly affected by the activated coil. It is worthy to note that the low pressure of the pump was because of the low conductivity of the saltwater solution.

Figure 11 shows the profile of the pressure variations in the longitudinal direction. The pressure changes after and before the pump. The red line area in Fig. 11 is the affected zone under the pump. As shown in Figs. 10 and 11, the pressure gradient changes by the pump, indicating the correct performance of the simulated model.

In Fig. 12, the velocity variations underneath the coils are shown three-dimensionally. As can be seen, the maximum velocity was underneath the middle coil. This indicated the concentration of the Lorentz force applied in

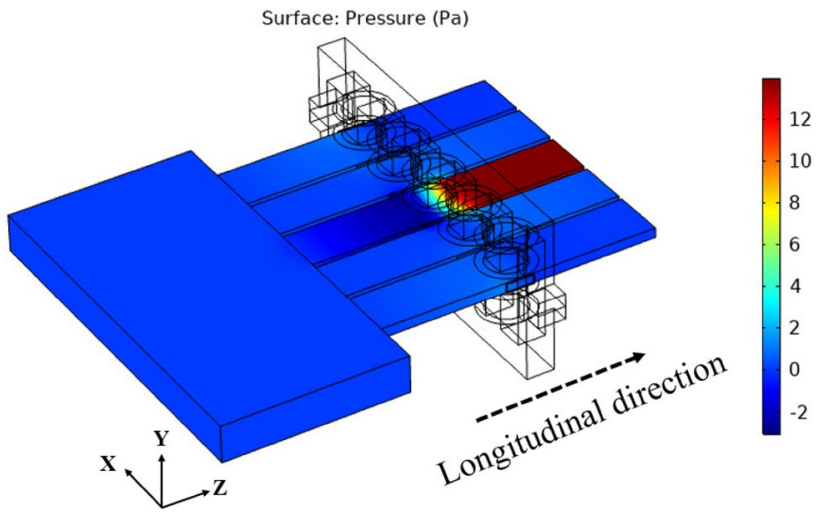

Fig. 10 The contour of the pressure variations in the middle array active mode and the closed channel end
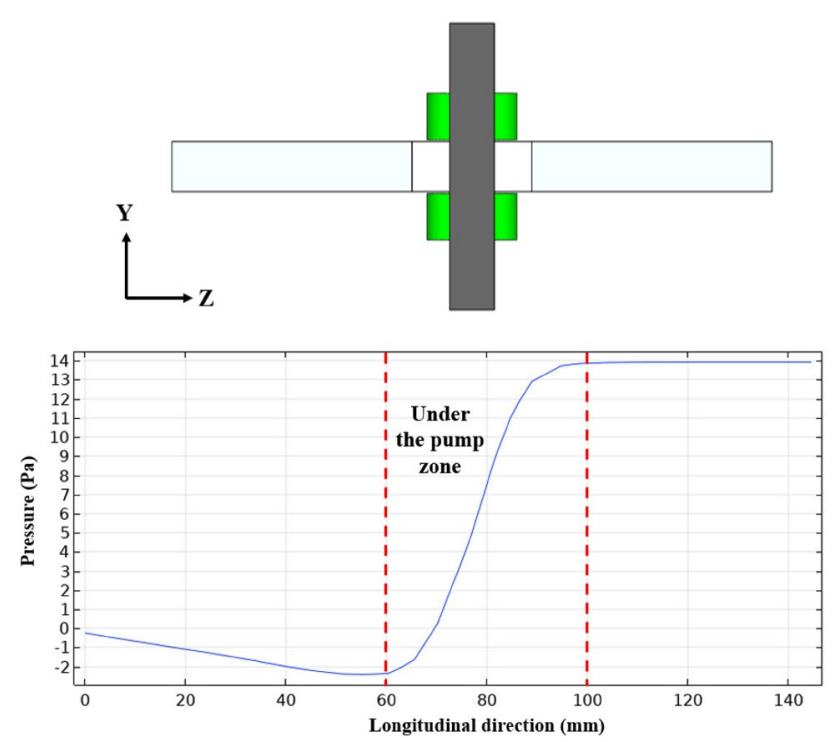

Fig. 11 The profile of the pressure variations in the simulation in longitudinal direction

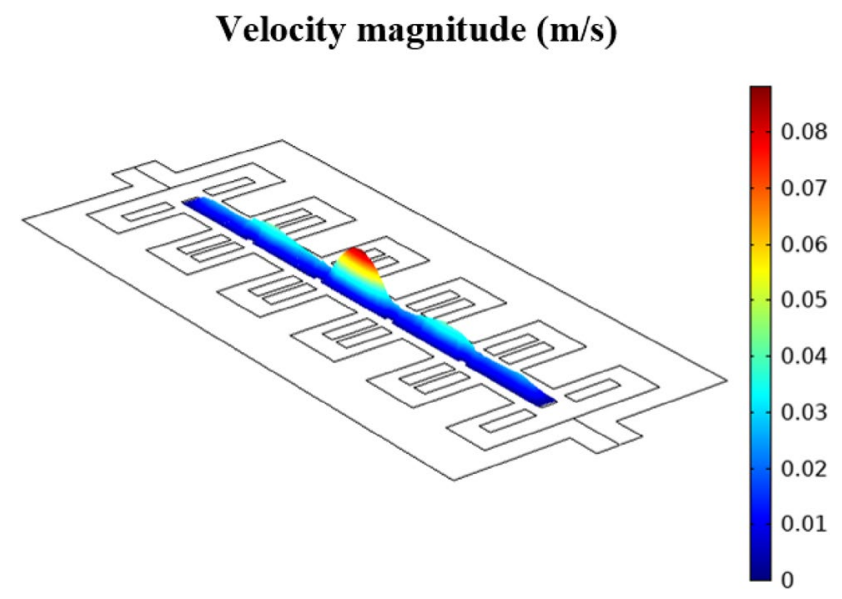

Fig. 12 The 3D contour of the velocity variations 


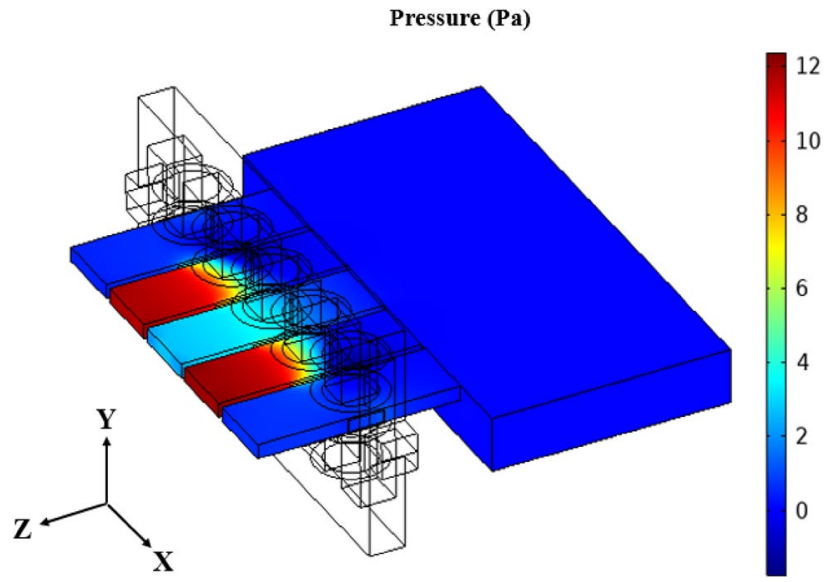

Fig.13 The pressure variations of the active electromagnets number 2 and 4
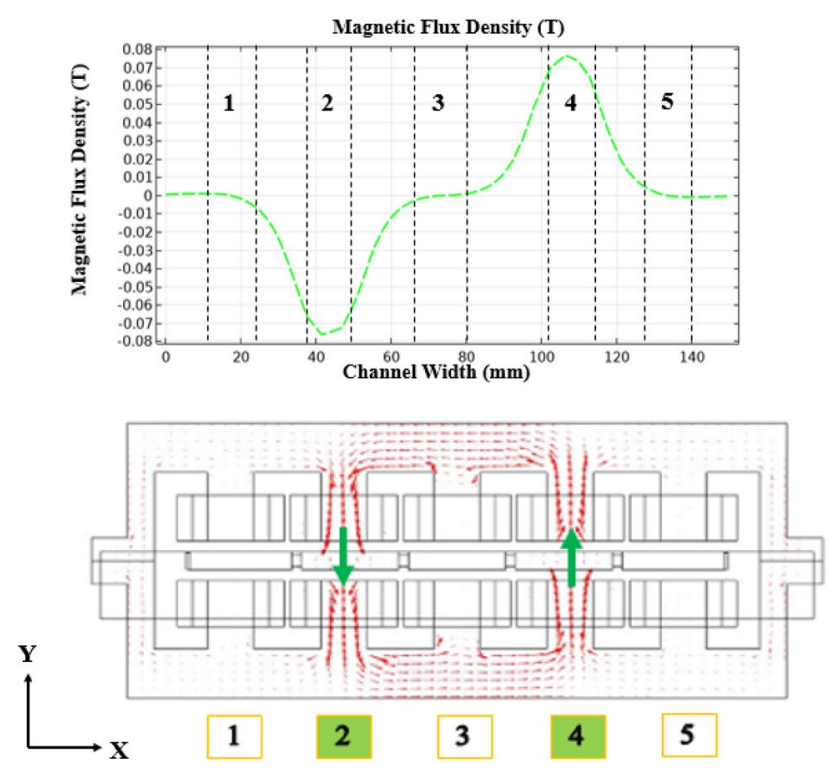

Fig. 14 The variations of contour and magnetic field lines of the active arrays number 2 and 4 in opposite directions

this area and confirmed the accuracy of the performance of the arrayed electromagnetic pump.

Figure 13 depicts the pressure variations in activating mode of the coils number 2 and 4 . Based on this figure, channels 2 and 4 are independent of each other; therefore, the pressure is separately imposed to each of these channels. As can be seen, the magnetic flux slightly affected channel number 3 , in which the pressure was nearly $3 \mathrm{~Pa}$. The pressure in both channels number 2 and 4 was equal to $12 \mathrm{~Pa}$.

Based on Fig. 14, the electromagnets number 2 and 4 are active, but the current directions of the coils are in opposite directions. In this case, the direction of the

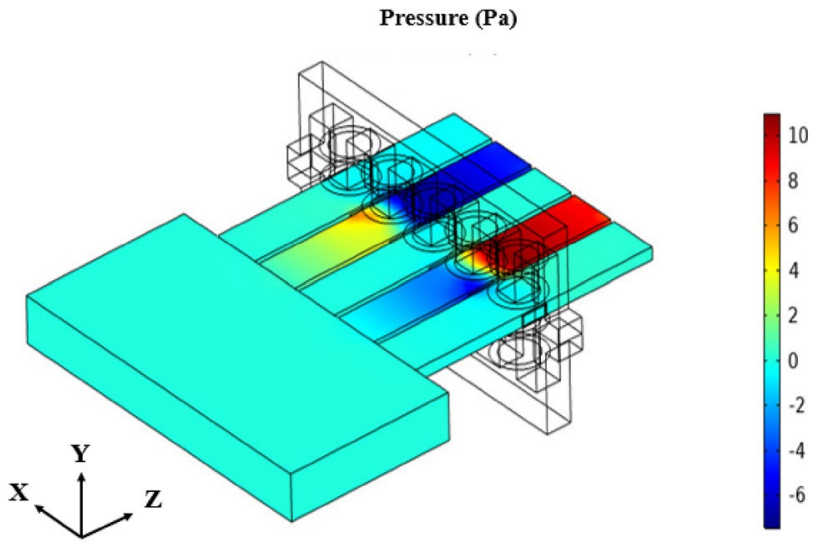

Fig. 15 The pressure variations of the active arrays number 2 and 4 in the opposite directions

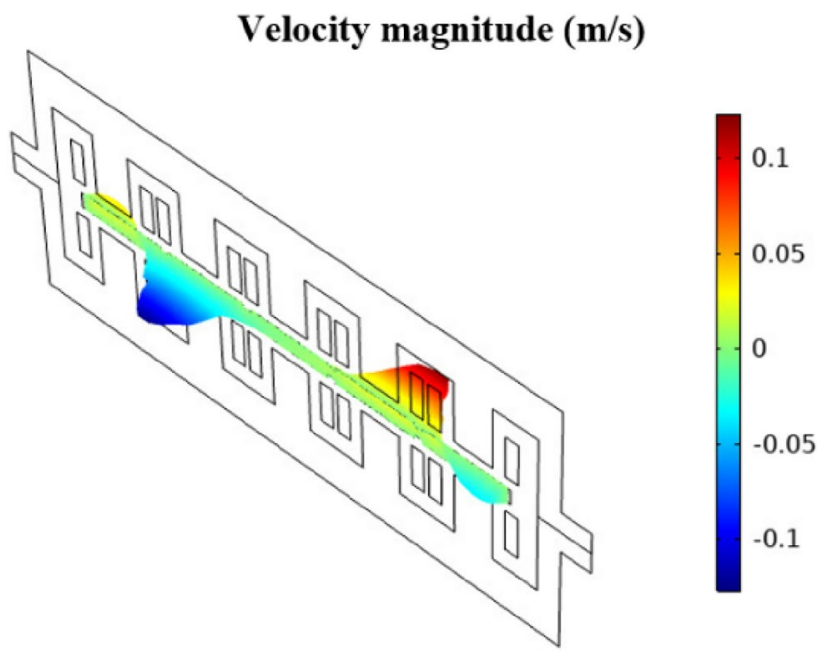

Fig. 16 The 3D contour of the velocity variations in the longitudinal direction of the active array pumps number 2 and 4 in opposite directions

magnetic fields in the channels had a $180^{\circ}$ difference. Due to this, the Lorentz force was applied in the opposite direction as well.

Figure 15 exhibits the pressure variations in this mode. As shown, the pressure changes in the two modes have a converse relationship. It shows the difference between the suction and head of the pump when two coils are activated in opposite directions.

Figure 16 represents the 3D variations of longitudinal component of the velocity underneath the electromagnets. The magnitudes of the velocity are equal, but in opposite directions, demonstrating that driving Lorentz forces can be generated in each subchannel, individually or separately.

In the last mode, all the arrays were activated simultaneously. Figure 17 illustrates the magnetic field lines and

\section{SN Applied Sciences}



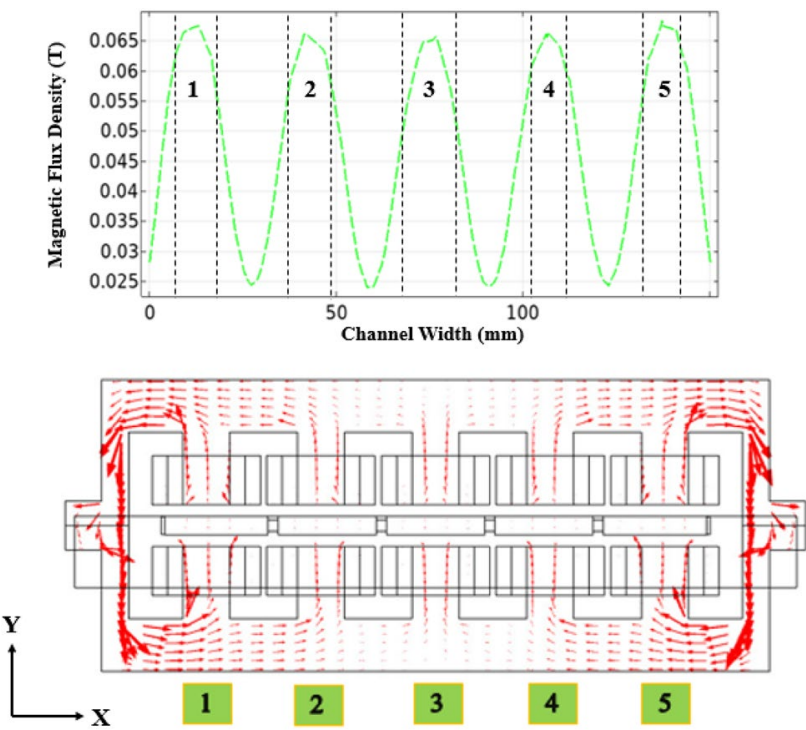

Fig. 17 The magnetic field lines and graph when all the arrays were active

its profile of variations in the transverse direction below the coils. As can be seen, an equal magnetic field is distributed under each coil across the channel.

According to Fig. 18, similar to regular electromagnetic pumps, the velocity variations on the sides are higher than those in the center; thus, the velocity profile is in the form of M-Shape.

\section{Experimental results}

To verify the simulation results, the arrayed electromagnetic pump was manufactured and tested experimentally. The experimental tests are presented in Fig. 19. It should be mentioned that the current of the coil was 3 amp during the experimental tests. Due to the low conductivity of water solutions, the pressure level of the pump was not sufficient to use any pressure gages. In order for the fluid to be constantly present under the electromagnets, the reservoir and the pump had an angle of $5^{\circ}$ with the horizon; this angle was also used for calculating the pressure of the pump. Figure 19 shows different combinations of coils activation during the experimental tests. The direction of the flow is indicated with black arrows. As shown in Fig. 19a, coil number 2 is activated and the water is pushed in this area and in other lines, the water line is constant. The bubbles around the electrodes are formed on account of the Electrochemical effect on the solution. Based on Fig. 19b, coil number 2 and 4 are activated and the water is pushed equally in these areas. As could be seen in Fig. $19 d$, when coils number 1,2 , and 3 are activated, the water could move only under these coils; this indicates that the velocity profile was created as desired. The effect of magnetic field direction is represented in Fig. 19e; as shown, coils number 2 and 4 are activated, but in coil number 2 , the polarity of magnetic excitation is reversed, which pushes the water backwards. The simulation of this state is shown in Fig. 16. It is noteworthy that the flow circuit was not closed and the flow did not come back to the channel from the head of the pump. Once the pump is working in the reverse direction, the pump pushes the flow back to the thank.

In order to calculate the pressure of the pump, the fluid pressure available in front of the pump was calculated. Figure 20 shows the angle of the reservoir with the horizon. In this case, the fluid was moved $1.5 \mathrm{~cm}$ forward thanks to the pump force.

The pump force was calculated considering the amount of the fluid displaced in front of the pump as follows:

$P=\rho g h \sin \left(5^{\circ}\right)=1000 \times 9.8 \times 1.5 \times 10^{-2} \times \sin \left(5^{\circ}\right)=12.8 p a$

When the arrays number 2 and 4 were active (Fig. 13), the pressure magnitude acquired in the simulation was $12 \mathrm{MPa}$. Therefore, the error of the simulation and the
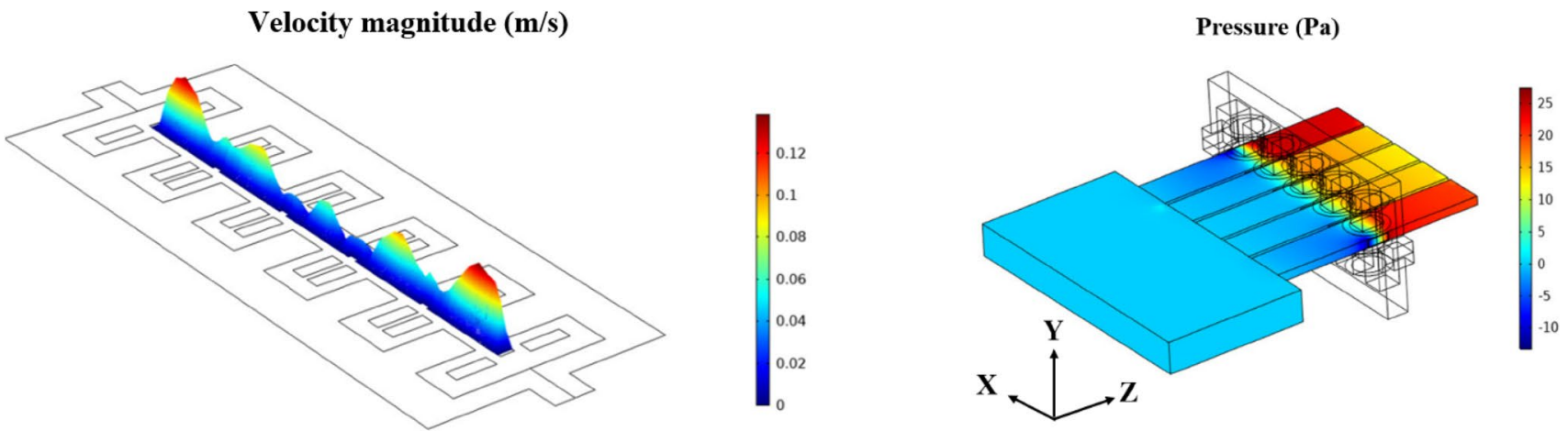

Fig. 18 The 3D contour of the pressure variations and velocity once all the arrays were active 


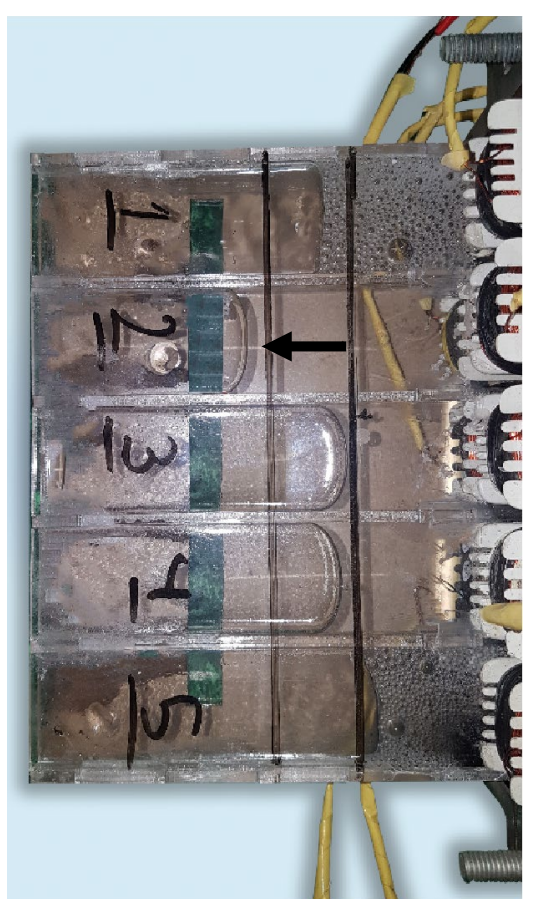

a number 2 is activated

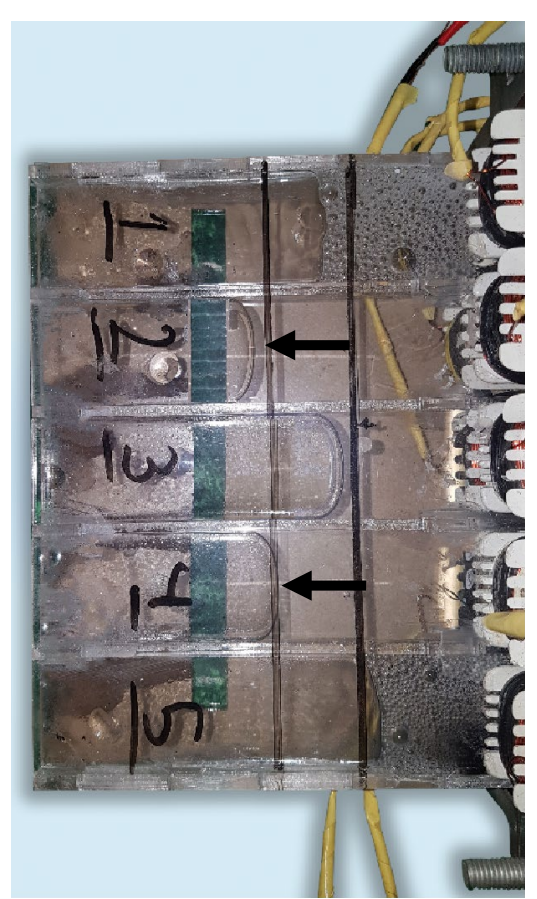

b number 2 and 4 are activated

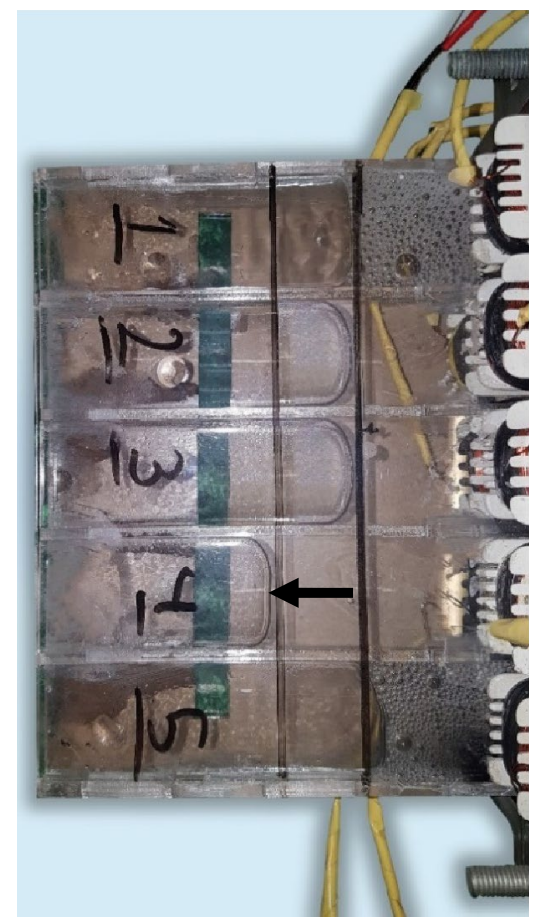

c number 4 is activated

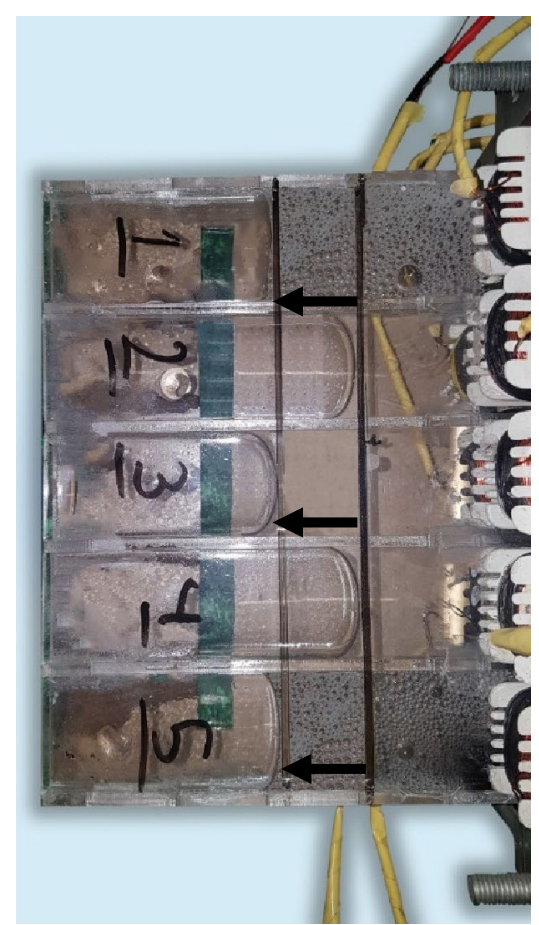

d number 1,3 and 5 are activated

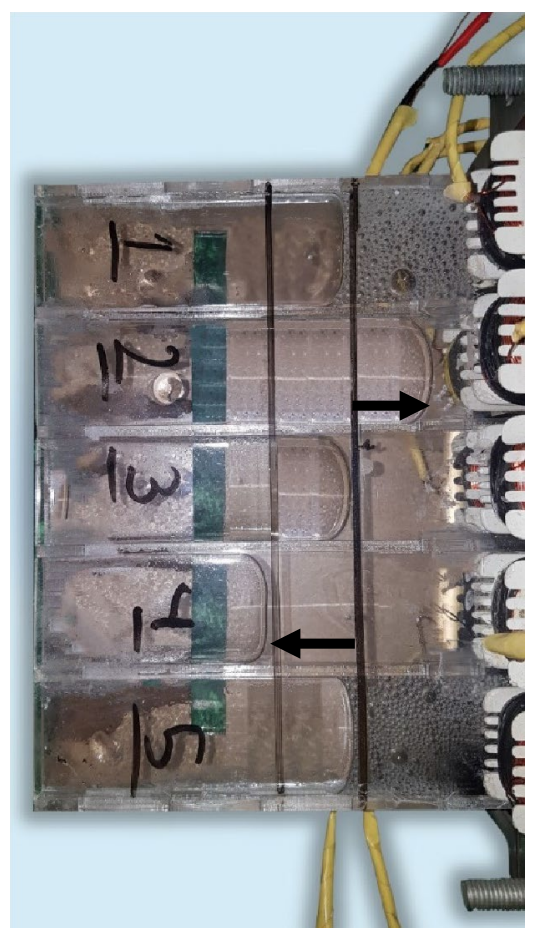

e number 2 (reverse) and 4 are activated

Fig. 19 The experimental test results 


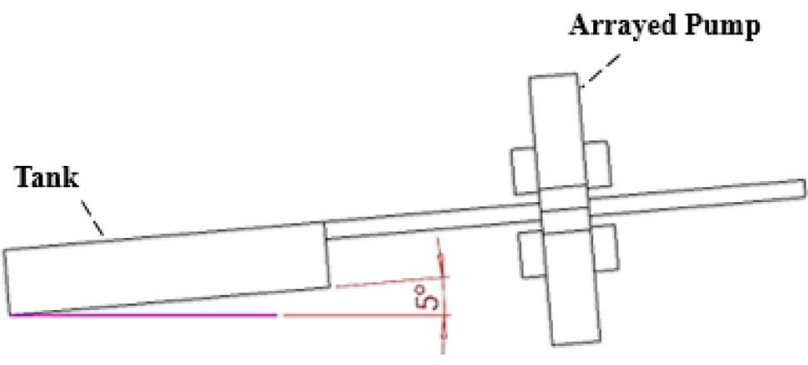

Fig. 20 The angle of the reservoir and pump with the horizon

experimental test equaled $6 \%$. To measure the fluid velocity, one of the arrays was first activated and the output fluid was separately collected in a container in the final part of the path. Subsequently, the fluid flow rate was obtained by measuring the gathered fluid and turning on the time of the pump. The linear velocity of the fluid was calculated through dividing the flow rate by the area of the channel cross-section. The fluid volume gathered at $20 \mathrm{~s}$ was equal to $12 \times 125 \times 45=67,500$. Hence, the volumetric flow rate equaled $Q=\frac{v}{t}=33751 \mathrm{exmm}^{3}-1 \mathrm{exs}$. The cross-section of the channel in the output path of the fluid was $2.5 \times 20 \mathrm{~mm}^{2}$. Consequently, the fluid linear velocity was equal to $u=\frac{Q}{A}=67.5 \mathrm{~mm} / \mathrm{s}=0.067 \mathrm{~m} / \mathrm{s}$. According to the fluid velocity calculated by the simulation $(0.08 \mathrm{~m} / \mathrm{s})$, a $16 \%$ error was observed. One of the sources of this error was the friction between the fluid and the wall, which was ignored in the simulation.

\section{Discussion}

As mentioned in the result sections, the novel arrayed electromagnetic pump structure could create any type of velocity and pressure profile, which is necessary in various applications. In other works, velocity profiles are not controllable and are highly influenced by the fluid and pump parameters $[10,18,21-23]$. Figure 21 a shows the velocity profile in different Hartman numbers (different fluids). As could be seen, the velocity was higher in the vicinity of the electrodes and had the minimum value in the center area of the channel. The magnitude of the velocity also changed with Hartman number. Not only did it change in width, but also it was not uniform in axial direction, as shown in Fig. $21 \mathrm{~b}$. Under the magnet area ( $x=0$ and $x=-1.54$ ), the biggest turbulence of velocity was found where the pump made Lorentz force move the fluid. Figure $21 \mathrm{c}$ demonstrates one of the arrayed electromagnetic pump modes. The velocity was positive in $40 \mathrm{~mm}$ of the channel width and was reversed in $110 \mathrm{~mm}$ of it. This feature could be employed in electromagnetic pump applications; for instance, it could be employed in EMT process in Die casting procedures and based on the shape of the sample, the velocity and pressure of the molten metal could be adjusted; therefore, all the parts of the mold would be filled.

\section{Conclusion}

In this research, an arrayed electromagnetic pump with the capability of controlling the magnetic field in the transverse direction of a rectangular channel was designed and manufactured. The designed arrayed pump was simulated in COMSOL and its different modes of excitations were investigated. The fluid used was $20 \% \mathrm{NaOH}$ solution. The magnetic field in each area was controllable utilizing coils. in addition, controlling the amount of the input current to them was conducive to taking control of the magnetic field. Hence, the pressure amount created in each part of the channel was under control; in fact, creating any arbitrary velocity profile in the channel width was possible. In the experimental test, different velocity profiles could be created by activating various electromagnets. On account of the low conductivity of the fluid, the pump pressure was $12 \mathrm{~Pa}$ equivalent to $1.5 \mathrm{~cm}$ of fluid at an angle of $5^{\circ}$. By measuring the fluid pumped per unit time, the flow rate and velocity of the fluid were calculated. Different velocity profiles were investigated in various conditions through simulation. Once all the arrays were active, the arrayed pump would be equivalent to the regular pumps and create an M-shape profile. Finally, it could be recommended 


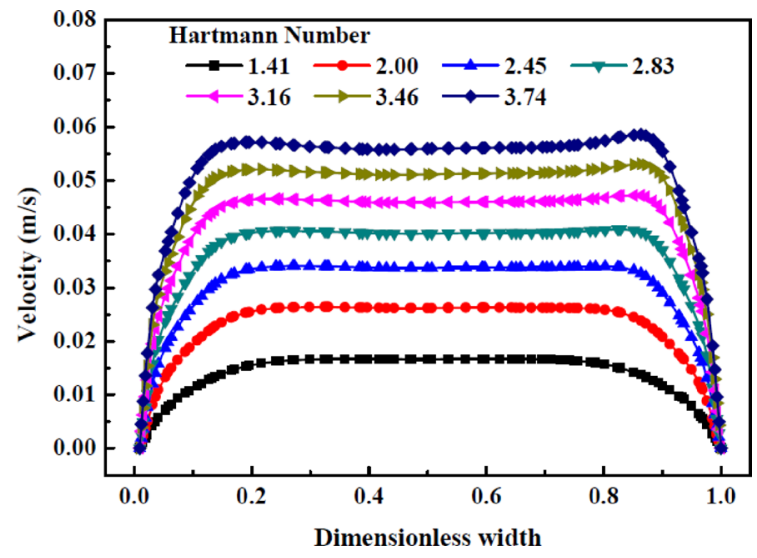

a Velocity variation with dimensionless width (Lee et al. [22])

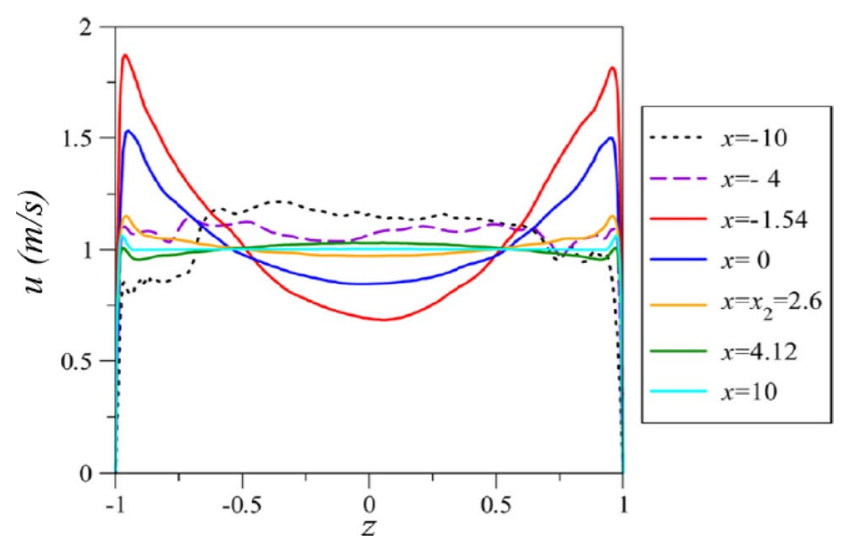

b Velocity Profiles in different axial positions (Klüber et al. [23])

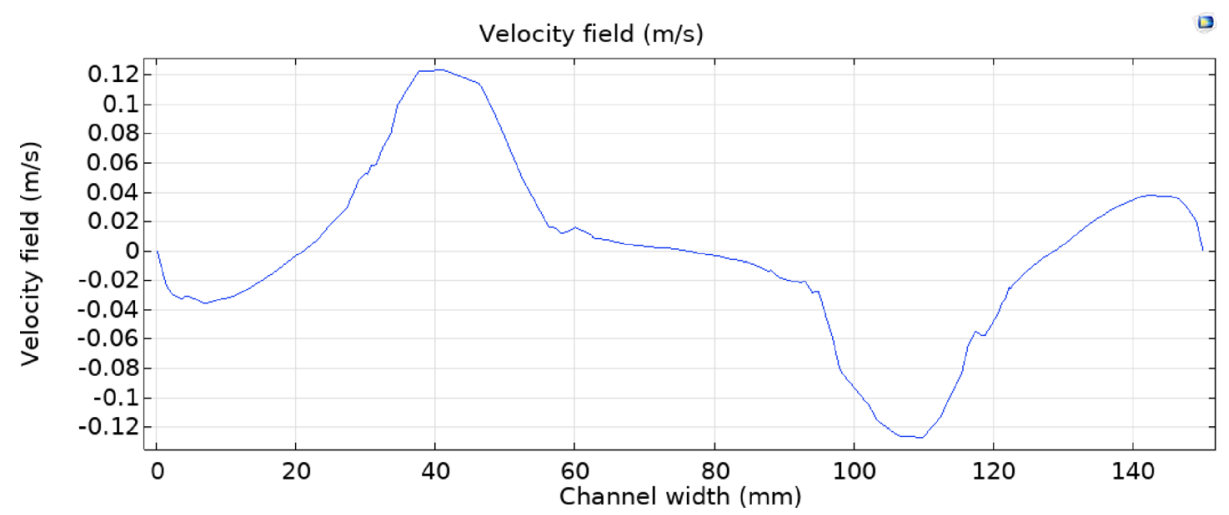

c Velocity profile while arrays number 2 and 4 are activated in contrary direction to each other (present study)

Fig. 21 Comparison of velocity profiles in other works to those in the present study

to test the array electromagnetic pump with molten metals which have high electrical conductivity.

\section{Declarations}

Conflict of interest On behalf of all authors, the corresponding author states that there is no conflict of interest.

Open Access This article is licensed under a Creative Commons Attribution 4.0 International License, which permits use, sharing, adaptation, distribution and reproduction in any medium or format, as long as you give appropriate credit to the original author(s) and the source, provide a link to the Creative Commons licence, and indicate if changes were made. The images or other third party material in this article are included in the article's Creative Commons licence, unless indicated otherwise in a credit line to the material. If material is not included in the article's Creative Commons licence and your intended use is not permitted by statutory regulation or exceeds the permitted use, you will need to obtain permission directly from the copyright holder. To view a copy of this licence, visit http://creativecommons. org/licenses/by/4.0/.

\section{References}

1. Gutierrez AU, Heckathorn CE (1965) Electromagnetic pumps for liquid metals. Naval Postgraduate School, Monterey

2. Al-Habahbeh O et al (2016) Review of magnetohydrodynamic pump applications. Alex Eng J 55(2):1347-1358

3. Wang L et al (2019) Experimental study and optimized design on electromagnetic pump for liquid sodium. Ann Nucl Energy 124:426-440

4. Ramos Jl, Winowich NS (1990) Finite difference and finite element methods for mhd channel flows. Int J Numer Methods Fluids 11(6):907-934

5. Hughes M, Pericleous KA, Cross M (1995) The numerical modelling of DC electromagnetic pump and brake flow. Appl Math Model 19(12):713-723

6. Andreev O, Kolesnikov Y, Thess A (2006) Experimental study of liquid metal channel flow under the influence of a nonuniform magnetic field. Phys Fluids 18(6):065108 
7. Votyakov EV, Zienicke E (2007) Numerical study of liquid metal flow in a rectangular duct under the influence of a heterogenous magnetic field. FDMP 3(2):97-114

8. Daoud A, Kandev N (2008) Magneto-hydrodynamic numerical study of DC electromagnetic pump for liquid metal. In: Proceedings of the COMSOL conference, Hannover, Germany

9. Aoki LP, Maunsell MG, Schulz HE (2012) A magnetohydrodynamic study of behavior in an electrolyte fluid using numerical and experimental solutions. Eng Térm 11:53-60

10. Aoki LP, Schulz HE, Maunsell MG (2013) An MHD study of the behavior of an electrolyte solution using 3D Numerical simulation and experimental results. In: Comsol Conference

11. Jamalabadi MA, Park JH (2014) Electro-magnetic ship propulsion stability under gusts. Int J Sci: Basic Appl Res 14:421-427

12. Dong $X$ et al (2013) 3D simulation of plane induction electromagnetic pump for the supply of liquid Alb. J Mater Process Technol 213:1426-1432

13. Dong $X$ et al (2015) Coupling analysis of the electromagnetic transport of liquid aluminum alloy during casting. J Mater Process Technol 222:197-205

14. Dong $X$ et al (2016) A liquid aluminum alloy electromagnetic transport process for high pressure die casting. J Mater Process Technol 234:217-227

15. Abdullina KI, Bogovalov SV (2015) 3-D numerical modeling of MHD flows in variable magnetic field. Phys Proced 72:351-357

16. Derakhshan S, Yazdani K (2016) 3D analysis of magnetohydrodynamic (MHD) micropump performance using numerical method. J Mech 32(1):55-62

17. Geza V, Nacke BJM (2016) Numerical simulation of core-free design of a large electromagnetic pump with double stator. Magnetohydrodynamics 52(3):287-301
18. Kwak J, Kim HR (2018) Design optimization analysis of a large electromagnetic pump for sodium coolant transportation in PGSFR. Ann Nucl Energy 121:62-67

19. Lee GH, Kim HR (2018) Numerical analysis of the electromagnetic force for design optimization of a rectangular direct current electromagnetic pump. Nucl Eng Technol 50(6):869-876

20. Wang $Y$ et al (2019) Experimental and numerical analysis on a compact liquid metal blade heat dissipator with twin stage electromagnetic pumps. Int Commun Heat Mass Transf 104:15-22

21. Azimi-Boulali J et al (2019) A study on the 3D fluid flow of MHD micropump. J Braz Soc Mech Sci Eng 41(11):1-15

22. Seo J-H et al (2020) Numerical investigations on magnetohydrodynamic pump based microchannel cooling system for heat dissipating element. Symmetry 12(10):1713

23. Klüber V, Bühler L, Mistrangelo C (2020) Numerical simulation of 3D magnetohydrodynamic liquid metal flow in a spatially varying solenoidal magnetic field. Fusion Eng Des 156:111659

24. Zhang X-D, Zhou Y-X, Liu JJATE (2020) A novel layered stack electromagnetic pump towards circulating metal fluid: design, fabrication and test. Appl Therm Eng 179:115

25. Foxboro I (1999) Table of conductivity vs. concentration for common solutions. https://www.academia.edu/17588429/Condu ctivity_v_Concentration

26. www.comsol.co.in/blogs/size-parameters-free-tetrahedralmeshing-comsol-multiphysics

Publisher's Note Springer Nature remains neutral with regard to jurisdictional claims in published maps and institutional affiliations. 